\title{
LINEAR RELATIVE CANONICAL ANALYSIS OF EUCLIDEAN RANDOM VARIABLES, ASYMPTOTIC STUDY AND SOME APPLICATIONS
}

\author{
JACQUES DaUXOIS ${ }^{1,2}$, GUY MARTIAL NKIET ${ }^{3}$ AND YVES ROMAIN ${ }^{1}$ \\ ${ }^{1}$ Laboratoire de Statistique et Probabilités, UMR CNRS C5583, Université Paul Sabatier, 118 Route \\ de Narbonne, F-31062 Toulouse Cedex 04, France, e-mail: jfdauxois@ifrance.com; \\ Yves.Romain@math.ups-tlse.fr \\ ${ }^{2}$ Equipe GRIMM, Université Toulouse Le Mirail, 31058 Toulouse cedex, France \\ ${ }^{3}$ Département de Mathématiques et Informatique, Université des Sciences et Techniques de Masuku, \\ BP943 Franceville, Gabon, e-mail: gnkiet@hotmail.com
}

(Received May 2, 2002; revised May 26, 2003)

\begin{abstract}
We introduce the Linear Relative Canonical Analysis (LRCA) of Euclidean random variables. Then similar properties than for usual linear Canonical Analysis are obtained. Furthermore, we develop an asymptotic study of LRCA and apply the obtained results to tests for lack of relative linear association, dimensionality and invariance.
\end{abstract}

Key words and phrases: Canonical analysis, relative canonical analysis, asymptotic study, linear relative association, invariance, additional information, (relative) canonical coefficient, partial canonical correlation.

\section{Introduction}

In some practical situations, one may have to make a statistical analysis on some variables when there is a noise variable. An approach for such situations consists in making the chosen analysis after removing the effect of this later variable; this is done by considering residuals of regression on that variable. This approach has led to known methods; an example can be found in discriminant analysis with a covariate which is known to have the same mean in the related groups (see e.g. Fujikoshi and Khatri (1990), Baccini et al. (2001)). For canonical analysis, the same approach gave partial (see Rao (1969)) part and bipartial (see Timm and Carlson (1976)) canonical correlation analysis. In this later work, statistical inferences based on the canonical coefficients related to these analyses were proposed. Although the obvious interest of these methods, it seems that there does not exist an extensive study of their properties as it is the case for usual Linear Canonical Analysis (LCA). Particularly, the aforementioned statistical inference shows the interest of making an asymptotic study of these analyses.

In this paper, we define the Linear Relative Canonical Analysis (LRCA) of Euclidean random variables and show that this analysis is in fact a LCA for suitable random variables and can be seen as a generalization of partial canonical correlation analysis. Then, some properties of LRCA are obtained from those of LCA. Next, we focus on the asymptotic study of LRCA. Although this analysis is a particular LCA, the results of asymptotic studies of LCA (see Arconte (1980), Pousse (1992), Anderson (1999), Fine (2000)) can not be applied because we do not have an i.i.d. sample of the related random 
variables. Indeed, these variables can not be observed since their definition involves covariance operators which are unknown in practice. So, we develop an asymptotic study for LRCA. The obtained results are used for defining tests for lack of relative linear association, dimensionality for LRCA, and invariance when the corresponding variables are transformed by linear maps (see the end of Section 2 for definition and properties and Section 4.3 for inference procedure).

\section{Linear relative canonical analysis}

Let $(\Omega, \mathcal{A}, P)$ be a probability space; along the paper we will work with random variables (r.v.) defined on $(\Omega, \mathcal{A}, P)$ and valued into Euclidean spaces (i.e. finite dimensional Hilbertian space). When $F$ is such a space, we will denote by $\langle\cdot, \cdot\rangle_{F}$ its inner product and by $\|\cdot\|_{F}$ the associated norm. We will use the usual tensor product $\otimes$ such that, for any vectors $u$ and $v$ belonging to Euclidean spaces $F$ and $G$ respectively, $u \otimes v$ is the linear map: $h \in F \mapsto\langle h, u\rangle_{F} v \in G$; if $u=v$ we will write $u^{\otimes^{2}}$ instead of $u \otimes u$. The properties of $\otimes$ (and other tensor products) and the related matrix expressions can be found in Dauxois et al. (1994). When $X$ is a random variable valued into an Euclidean space $\mathcal{X}$ and satisfying $\mathbb{E}\left(\|X\|_{\mathcal{X}}^{2}\right)<+\infty$, we will denote by $L_{X}$ the linear map: $u \in \mathcal{X} \mapsto\langle u, X\rangle_{\mathcal{X}} \in L^{2}(\Omega, \mathcal{A}, P)$. For all operator $T$, we will denote by $T^{*}$ its adjoint. It is easily seen that $L_{X}^{*}$ is the map: $Z \in L^{2}(\Omega, \mathcal{A}, P) \mapsto \mathbb{E}(Z X) \in \mathcal{X}$; thus if $X$ is centered, its covariance operator $V_{X}:=\mathbb{E}\left(X^{\otimes^{2}}\right)$ verifies $V_{X}=L_{X}^{*} L_{X}$.

For $m \in\{1,2,3\}$, let us consider a centered r.v. $X_{m}$ defined on $(\Omega, \mathcal{A}, P)$ and valued into a Euclidean space $\mathcal{X}_{m}$ with dimension $p_{m}$; without loss of generality we assume that $p_{1} \leq p_{2}$. Further, we suppose that $\mathbb{E}\left(\left\|X_{m}\right\|_{\mathcal{X}_{m}}^{2}\right)<+\infty$ and we define

$$
E_{m}:=R\left(L_{X_{m}}\right)
$$

where $R(T)$ denotes the range of the operator $T$, and for $k \in\{1,2\}$

$$
E_{k \cdot 3}:=\left(E_{k}+E_{3}\right) \Theta E_{3}
$$

where $\ominus$ denotes the orthogonal difference defined by: $E \ominus F=E \cap F^{\perp}$ where $E$ and $F$ are Euclidean spaces such that $F \subset E$. Notice that, denoting by $\Pi_{E}$ the orthogonal projection operator onto the subspace $E$ and by $E^{\perp}$ its orthogonal space, one has

$$
E_{k \cdot 3}=\Pi_{E_{3}^{-}}\left(E_{k}\right)
$$

Now, we define:

Definition 2.1. The linear relative canonical analysis (LRCA) of $X_{1}$ and $X_{2}$ relative to $X_{3}$ is the canonical analysis (CA) of $E_{1 \cdot 3}$ and $E_{2 \cdot 3}$.

Notice that since $E_{1 \cdot 3}$ and $E_{2 \cdot 3}$ are finite-dimensional spaces, the previous CA is a linear CA (LCA) of random vectors (see Dauxois and Pousse (1975), Dauxois and Nkiet $(1997 a))$. When these spaces are $\mathbb{R}^{p}$-type ones, the components of these random vectors are r.v. which span the aforementioned spaces. In the more general framework of Euclidean spaces, these random vectors (say $X_{1 \cdot 3}$ and $X_{2 \cdot 3}$ ) are such that $E_{1 \cdot 3}=$ $R\left(L_{X_{1.3}}\right)$ and $E_{2 \cdot 3}=R\left(L_{X_{2,3}}\right)$. Now, we will search such random vectors. In order 
to simplify notation, we will write $L_{m}$ (resp. $V_{m}$ ) instead of $L_{X_{m}}$ (resp. $V_{X_{m}}$ ). Let us consider

$$
V_{m j}:=L_{m}^{*} L_{j}=\mathbb{E}\left(X_{j} \otimes X_{m}\right) \quad \text { for } \quad(m, j) \in\{1,2,3\}^{2} \quad \text { with } \quad m \neq j
$$

and put

$$
\begin{aligned}
& L_{k \cdot 3}:=L_{k}-L_{3} V_{3}^{\dagger} V_{3 k} \\
& X_{k \cdot 3}:=X_{k}-V_{k 3} V_{3}^{\dagger} X_{3} \quad(k=1,2),
\end{aligned}
$$

where $T^{\dagger}$ denotes the Moore-Penrose inverse of the operator $T$. We obtain

Lemma 2.1. For $k \in\{1,2\}$, one has:

(i) $E_{k \cdot 3}=R\left(L_{k \cdot 3}\right)$;

(ii) $L_{k \cdot 3}=L_{X_{k \cdot 3}}$.

Proof. (i) From equation (2.3), we have $u \in E_{k \cdot 3}$ if, and only if, there exists $x \in E_{k}$ such that $u=\Pi_{E_{3}} x=x-\Pi_{E_{3}} x$. Thus, from the equality $\Pi_{E_{3}}=L_{3} V_{3}^{\dagger} L_{3}^{*}$ and equation (2.1), $u \in E_{k \cdot 3}$ is equivalent to the existence of a vector $\alpha \in \mathcal{X}_{k}$ such that $u=L_{k} \alpha-L_{3} V_{3}^{\dagger} L_{3}^{*} L_{k} \alpha$, that is $u \in R\left(L_{k \cdot 3}\right)$.

(ii) For all $\alpha \in \mathcal{X}_{k}$, we have: $L_{k \cdot 3} \alpha=\left\langle\alpha, X_{k}\right\rangle_{\mathcal{X}_{k}}-\left\langle V_{3}^{\dagger} V_{3 k} \alpha, X_{3}\right\rangle_{\mathcal{X}_{3}}=\left\langle\alpha, X_{k}-\right.$ $\left.V_{k 3} V_{3}^{\dagger} X_{3}\right\rangle_{\mathcal{X}_{k}}=L_{X_{k \cdot 3}} \alpha ;$ this proves the lemma.

Since $E_{k \cdot 3}=R\left(L_{X_{k \cdot 3}}\right)$, we can state:

Proposition 2.1. The LRCA of $X_{1}$ and $X_{2}$ relative to $X_{3}$ is the LCA of the random variables $X_{1 \cdot 3}=X_{1}-V_{13} V_{3}^{\dagger} X_{3}$ and $X_{2 \cdot 3}=X_{2}-V_{23} V_{3}^{\dagger} X_{3}$.

Let us consider the operators:

$$
\begin{aligned}
& V_{k \cdot 3}=L_{k \cdot 3}^{*} L_{k \cdot 3}=\mathbb{E}\left(X_{k \cdot 3}^{\otimes^{2}}\right), \\
& V_{k m \cdot 3}=L_{k \cdot 3}^{*} L_{m \cdot 3}=\mathbb{E}\left(X_{m \cdot 3} \otimes X_{k \cdot 3}\right) \quad \text { for } \quad m \neq k
\end{aligned}
$$

we have:

$$
\begin{aligned}
V_{k \cdot 3} & =\left(L_{k}^{*}-V_{k 3} V_{3}^{\dagger} L_{3}^{*}\right)\left(L_{k}-L_{3} V_{3}^{\dagger} V_{3 k}\right) \\
& =V_{k}-V_{k 3} V_{3}^{\dagger} V_{3 k}
\end{aligned}
$$

and for $m \neq k$ :

$$
\begin{aligned}
V_{k m \cdot 3} & =\left(L_{k}^{*}-V_{k 3} V_{3}^{\dagger} L_{3}^{*}\right)\left(L_{m}-L_{3} V_{3}^{\dagger} V_{3 m}\right) \\
& =V_{k m}-V_{k 3} V_{3}^{\dagger} V_{3 m} .
\end{aligned}
$$

We deduce from the classical theory of LCA that the LRCA of $X_{1}$ and $X_{2}$ relative to $X_{3}$ is obtained for example from the spectral analysis of the selfadjoint operator

$$
T_{1 \cdot 3}=\left(V_{1 \cdot 3}^{\dagger}\right)^{1 / 2} V_{12 \cdot 3} V_{2 \cdot 3}^{\dagger} V_{21 \cdot 3}\left(V_{1 \cdot 3}^{\dagger}\right)^{1 / 2}
$$


where $T^{1 / 2}$ denotes the square root of a nonnegative operator $T$.

Remark 2.1. When $V_{1 \cdot 3}$ and $V_{2 \cdot 3}$ are invertible the spectral analysis of $T_{1 \cdot 3}$ is equivalent to that of $T_{1 \cdot 3}^{\prime}=V_{1 \cdot 3}^{-1} V_{12 \cdot 3} V_{2 \cdot 3}^{-1} V_{21 \cdot 3}$. That is known in the literature as partial canonical correlation analysis (see e.g. Rao (1969), Timm and Carlson (1976)). Then this last analysis appears as a particular case of the general relative canonical analysis of subspaces (see Dauxois and Nkiet (2002), Dauxois et al. (2004)), obtained by considering subspaces generated by specific linear functions of the original variables. In order to show up this property we prefer to use the terminology linear relative canonical analysis instead of partial canonical analysis. Notice that the part and bipartial canonical correlation analysis developed by Timm and Carlson (1976) can be reobtained from our framework by considering the CA of $E_{1}$ and $E_{2 \cdot 3}$, and $E_{1 \cdot 3}$ and $E_{2.4}$ respectively, where $E_{2.4}$ is constructed as in equation (2.2) with another Euclidean r.v. $X_{4}$.

The properties of LRCA are deduced from those of LCA. Hence the LRCA of $X_{1}$ and $X_{2}$ is characterized by a triple

$$
\left\{\left(\rho_{i}\right)_{0 \leq i \leq r},\left(\alpha_{1 \cdot 3}^{(i)}\right)_{0 \leq i \leq p_{1}},\left(\alpha_{2 \cdot 3}^{(i)}\right)_{0 \leq i \leq p_{2}}\right\}
$$

(where $r$ denotes the rank of $T_{1 \cdot 3}$ and $p_{k}$ is the dimension of $\mathcal{X}_{k}, k=1,2$ ) satisfying:

(P1) for each $i \in\{1, \ldots, r\}, \rho_{i}^{2}$ is the $i$-th greatest eigenvalue of $T_{1.3}$ and satisfies $0<\rho_{i} \leq \rho_{i-1} \leq 1$ (with $\rho_{0}=1$ );

(P2) the system $\left(\alpha_{1 \cdot 3}^{(i)}\right)_{0 \leq i \leq p_{1}}$ is an orthonormal basis of $\mathcal{X}_{1}$ such that each $\alpha_{1 \cdot 3}^{(i)}$ is an eigenvector of $T_{1 \cdot 3}$ verifying:

- if $i \leq r$, then $\alpha_{1.3}^{(i)}$ is associated with $\rho_{i}^{2}$

- if $i>r$, then $\alpha_{1.3}^{(i)}$ is associated with 0 ;

(P3) the system $\left(\alpha_{2.3}^{(i)}\right)_{0 \leq i \leq p_{1}}$ is an orthonormal basis of $\mathcal{X}_{2}$ such that:

- if $i \leq r$, then $\alpha_{2 \cdot 3}^{(i)}=\rho_{i}^{-1}\left(V_{2 \cdot 3}^{\dagger}\right)^{1 / 2} V_{21 \cdot 3}\left(V_{1 \cdot 3}^{\dagger}\right)^{1 / 2} \alpha_{1 \cdot 3}^{(i)}$; this equality is equivalent to $\alpha_{1 \cdot 3}^{(i)}=\rho_{i}^{-1}\left(V_{1 \cdot 3}^{\dagger}\right)^{1 / 2} V_{12 \cdot 3}\left(V_{2 \cdot 3}^{\dagger}\right)^{1 / 2} \alpha_{2 \cdot 3}^{(i)}$ and then $\alpha_{2 \cdot 3}^{(i)}$ is an eigenvector of $T_{2 \cdot 3}:=$ $\left(V_{2 \cdot 3}^{\dagger}\right)^{1 / 2} V_{21 \cdot 3} V_{1 \cdot 3}^{\dagger} V_{12 \cdot 3}\left(V_{2 \cdot 3}^{\dagger}\right)^{1 / 2}$ associated with $\rho_{i}^{2}$,

- if $i>r$, then $\alpha_{2 \cdot 3}^{(i)}$ is an eigenvector of $T_{2.3}$ associated with 0 .

The $\rho_{i}$ 's are termed the (relative) canonical coefficients associated to the LCRA; the (relative) canonical variates are the random variables defined for $(k, i) \in\{1,2\} \times$ $\left\{0, \ldots, p_{k}\right\}$ by:

$$
f_{k \cdot 3}^{(i)}:=L_{k \cdot 3}\left(V_{k \cdot 3}^{\dagger}\right)^{1 / 2} \alpha_{k \cdot 3}^{(i)}=\left\langle X_{k \cdot 3},\left(V_{k \cdot 3}^{\dagger}\right)^{1 / 2} \alpha_{k \cdot 3}^{(i)}\right\rangle \chi_{k} .
$$

Clearly, one has

$$
\left(V_{k \cdot 3}^{\dagger}\right)^{1 / 2} X_{k \cdot 3}=\sum_{i=1}^{p_{k}} f_{k \cdot 3}^{(i)} \alpha_{k \cdot 3}^{(i)}
$$

and

$$
\begin{aligned}
\mathbb{E}\left(f_{1 \cdot 3}^{(i)} f_{2 \cdot 3}^{(j)}\right) & =\left\langle L_{1 \cdot 3}\left(V_{1 \cdot 3}^{\dagger}\right)^{1 / 2} \alpha_{1 \cdot 3}^{(i)}, L_{2 \cdot 3}\left(V_{2 \cdot 3}^{\dagger}\right)^{1 / 2} \alpha_{2 \cdot 3}^{(j)}\right\rangle \\
& =\left\langle\alpha_{1 \cdot 3}^{(i)},\left(V_{1 \cdot 3}^{\dagger}\right)^{1 / 2} L_{1 \cdot 3}^{*} L_{2 \cdot 3}\left(V_{2 \cdot 3}^{\dagger}\right)^{1 / 2} \alpha_{2 \cdot 3}^{(j)}\right\rangle_{\mathcal{X}_{1}} \\
& =\left\langle\alpha_{1 \cdot 3}^{(i)},\left(V_{1 \cdot 3}^{\dagger}\right)^{1 / 2} V_{12 \cdot 3}\left(V_{2 \cdot 3}^{\dagger}\right)^{1 / 2} \alpha_{2 \cdot 3}^{(j)}\right\rangle_{\mathcal{X}_{1}}
\end{aligned}
$$


If $j>r, \alpha_{2 \cdot 3}^{(j)}$ belongs to the kernel of $T_{2 \cdot 3}$, that is also the kernel of $\left(V_{1 \cdot 3}^{\dagger}\right)^{1 / 2} V_{12 \cdot 3}\left(V_{2 \cdot 3}^{\dagger}\right)^{1 / 2}$; hence we have $\mathbb{E}\left(f_{1 \cdot 3}^{(i)} f_{2.3}^{(j)}\right)=0$. If $j \leq r$ then the use of (P3) and the orthonormality of the $\alpha_{1 \cdot 3}^{(i)}$ 's gives $\mathbb{E}\left(f_{1 \cdot 3}^{(i)} f_{2 \cdot 3}^{(j)}\right)=\rho_{j} \delta_{i j}$, where $\delta_{i j}$ denotes the Kronecker delta. Consequently, taking $\rho_{i}=0$ for $i>r$, we have for any $(i, j) \in\left\{0, \ldots, p_{1}\right\} \times\left\{0, \ldots, p_{2}\right\}$

$$
\mathbb{E}\left(f_{1 \cdot 3}^{(i)} f_{2 \cdot 3}^{(j)}\right)=\rho_{j} \delta_{i j} .
$$

Moreover, when for $k \in\{1,2\}, V_{k \cdot 3}$ is invertible, one has for $(i, j) \in\left\{1, \ldots, p_{k}\right\}^{2}$

$$
\begin{aligned}
\mathbb{E}\left(f_{k \cdot 3}^{(i)} f_{k \cdot 3}^{(j)}\right) & =\left\langle L_{k \cdot 3} V_{k \cdot 3}^{-1 / 2} \alpha_{k \cdot 3}^{(i)}, L_{k \cdot 3} V_{k \cdot 3}^{-1 / 2} \alpha_{k \cdot 3}^{(j)}\right\rangle \\
& =\left\langle\alpha_{k \cdot 3}^{(i)}, V_{k \cdot 3}^{-1 / 2} L_{k \cdot 3}^{*} L_{k \cdot 3} V_{k \cdot 3}^{-1 / 2} \alpha_{k \cdot 3}^{(j)}\right\rangle_{\mathcal{X}_{k}} \\
& =\left\langle\alpha_{k \cdot 3}^{(i)}, \alpha_{k \cdot 3}^{(j)}\right\rangle_{\mathcal{X}_{1}}=\delta_{i j} .
\end{aligned}
$$

In order to simplify the previous expressions, conditions for the invertibility of $V_{k \cdot 3}$ $(k=1,2)$ may be searched. They can be obtained from the following properties:

Lemma 2.2. For $k \in\{1,2\}$ :

(i) $E_{k} \cap E_{3}=L_{k}\left(\operatorname{ker}\left(V_{k \cdot 3}\right)\right)$;

(ii) $E_{k} \cap E_{3}=\{0\} \Leftrightarrow \operatorname{ker}\left(V_{k}\right)=\operatorname{ker}\left(V_{k \cdot 3}\right)$.

Proof. (i) Let $u$ be an element of $E_{k} \cap E_{3}$, then $\Pi_{E_{k}} \Pi_{E_{3}} u=u$ and there exists a vector $\alpha$ which can be chosen in $R\left(L_{k}^{*}\right)$ (because $\mathcal{X}_{k}=\operatorname{ker} L_{k} \oplus R\left(L_{k}^{*}\right)$ ) such that $u=L_{k} \alpha$. Since $\Pi_{E_{m}}=L_{m} V_{m}^{\dagger} L_{m}^{*}(m \in\{1,2,3\})$, we then have $L_{k} V_{k}^{\dagger} V_{k 3} V_{3}^{\dagger} V_{3 k} \alpha=L_{k} \alpha$. Premultiplying both sides of this equality by $L_{k}^{*}$ gives $\Pi_{R\left(V_{k}\right)} V_{k 3} V_{3}^{\dagger} V_{3 k} \alpha=V_{k} \alpha$; since $R\left(V_{k 3}\right)=R\left(L_{k}^{*} L_{3}\right) \subset R\left(L_{k}^{*}\right)=R\left(V_{k}\right)$, by equation (2.5) we obtain $V_{k \cdot 3} \alpha=0$. Hence $u \in L_{k}\left(\operatorname{ker}\left(V_{k \cdot 3}\right)\right)$ and thus $E_{k} \cap E_{3} \subset L_{k}\left(\operatorname{ker}\left(V_{k \cdot 3}\right)\right)$. Reciprocally, for all $\alpha \in \operatorname{ker}\left(V_{k \cdot 3}\right)$, one has $V_{k} \alpha=V_{k 3} V_{3}^{\dagger} V_{3 k} \alpha=L_{k}^{*} \Pi_{E_{3}} L_{k} \alpha$. Premultiplying both sides of this equality by $L_{k} V_{k}^{\dagger}$ and noticing that $L_{k} \Pi_{R\left(V_{k}\right)}=L_{k} \Pi_{R\left(L_{k}^{*}\right)}=L_{k}$ permit to obtain $u=\Pi_{E_{k}} \Pi_{E_{3}} u$, where $u=L_{k} \alpha$. Thus $u \in E_{k} \cap E_{3}$ and this proves that $L_{k}\left(\operatorname{ker}\left(V_{k \cdot 3}\right)\right) \subset E_{k} \cap E_{3}$.

(ii) If $E_{k} \cap E_{3}=\{0\}$ then, from (i), $\alpha \in \operatorname{ker}\left(V_{k \cdot 3}\right)$ implies $L_{k} \alpha=0$, that is $\alpha \in$ $\operatorname{ker}\left(L_{k}\right)=\operatorname{ker}\left(V_{k}\right)$. Reciprocally, if $\operatorname{ker}\left(V_{k}\right)=\operatorname{ker}\left(V_{k \cdot 3}\right)$ then from (i), we have $E_{k} \cap E_{3}=$ $L_{k}\left(\operatorname{ker}\left(V_{k}\right)\right)=L_{k}\left(\operatorname{ker}\left(L_{k}\right)\right)=\{0\}$.

From this lemma we deduce that, for $k \in\{1,2\}$, if $V_{k}$ is invertible and $E_{k} \cap E_{3}=$ $\{0\}$ then $V_{k \cdot 3}$ is invertible. These are sufficient but not necessary conditions for the invertibility of $V_{k \cdot 3}$.

Remark 2.2. When, for $m \in\{1,2,3\}$, a basis is chosen in $\mathcal{X}_{m}$ :

1) the invertibility of $V_{m}$ is equivalent to the linear (algebraic) independence of the components of $X_{m}$ related to the basis of $\mathcal{X}_{m}$ which is considered. One can always reduce to that situation by removing some of these components. In the literature, $V_{m}$ is often supposed to be invertible and it is admitted that this assumption does not restrict the generality;

2) when the $V_{m}$ 's are invertible, the condition $E_{k} \cap E_{3}=\{0\}(k=1,2)$ means that the system made up by the components of $X_{k}$ additioned to that of $X_{3}$ is linearly independent. That situation can always be obtained by removing some components in $X_{k}$ and/or in $X_{3}$; 
Our approach consists in defining LRCA by using the CA of Euclidean spaces (see Dauxois and Nkiet (1997a)); one of the interests of this approach is that it permits to see several classical methods as particular cases of this CA. Two examples are given below.

Example 2.1, LCA with linear constraints. This method (see Yanai and Takane (1992)) consists in searching canonical variates of $X_{1}$ and $X_{2}$ having the form $f_{k}^{(i)}=$ $\left\langle\alpha_{k}^{(i)}, X_{k}\right\rangle_{\mathcal{X}_{k}}$ with the linear constraints $A_{k} \alpha_{k}^{(i)}=0$, where $A_{k}$ is a linear map from $\mathcal{X}_{k}$ to another Euclidean space $\mathcal{X}_{k}^{\prime}(k=1,2)$. Suppose that, for $k \in\{1,2\}, V_{k}$ is invertible, and put $Y_{k}:=A_{k} V_{k}^{-1} X_{k}$ and $E_{Y_{k}}:=R\left(L_{Y_{k}}\right)$. It is clear that $E_{Y_{k}} \subset E_{k}$; then $\left(E_{k}+E_{Y_{k}}\right) \ominus E_{Y_{k}}=E_{k} \ominus E_{Y_{k}}$. For any $\alpha \in \mathcal{X}_{k}$ and any $\beta \in \mathcal{X}_{k}^{\prime}$, one has

$$
\mathbb{E}\left(\left\langle\alpha, X_{k}\right\rangle_{\mathcal{X}_{k}}\left\langle\beta, Y_{k}\right\rangle_{\mathcal{X}_{k}^{\prime}}\right)=\left\langle\mathbb{E}\left(X_{k} \otimes Y_{k}\right) \alpha, \beta\right\rangle_{\mathcal{X}_{k}^{\prime}}=\left\langle A_{k} \alpha, \beta\right\rangle_{\mathcal{X}_{k}^{\prime}}
$$

consequently, a r.v. $f:=\left\langle\alpha, X_{k}\right\rangle_{\mathcal{X}_{k}}$ belongs to $E_{k} \ominus E_{Y_{k}}$ if, and only if, for any $\beta \in$ $\mathcal{X}_{k}^{\prime},\left\langle A_{k} \alpha, \beta\right\rangle_{\mathcal{X}_{k}^{\prime}}=0$; that is $A_{k} \alpha=0$. Consequently, the LCA of $X_{1}$ and $X_{2}$ with the previous linear constraints is the CA of $E_{1} \ominus E_{Y_{1}}$ and $E_{2} \ominus E_{Y_{2}}$. Now, it will be reformulated as LCA for suitably transformed variates. For $k \in\{1,2\}$, from Lemma 2.1 we have $E_{k} \ominus E_{Y_{k}}=R\left(L_{Z_{k}}\right)$, where $Z_{k}:=X_{k}-U_{k} W_{k}^{\dagger} Y_{k}$ with $U_{k}:=\mathbb{E}\left(Y_{k} \otimes X_{k}\right)$ and $W_{k}:=\mathbb{E}\left(Y_{k}^{\otimes^{2}}\right)$. Therefore, the previous $\mathrm{CA}$ is the LCA of the random vectors $Z_{1}$ and $Z_{2}$ which can easily be expressed as transformed variates from $X_{1}$ and $X_{2}$ respectively. Indeed, from $U_{k}=\mathbb{E}\left(X_{k}^{\otimes^{2}}\right) V_{k}^{-1} A_{k}^{*}=A_{k}^{*}$ and $W_{k}=A_{k} V_{k}^{-1} \mathbb{E}\left(X_{k}^{\otimes^{2}}\right) V_{k}^{-1} A_{k}^{*}=A_{k} V_{k}^{-1} A_{k}^{*}$, we obtain $Z_{k}=\left(I_{k}-C_{k}\right) X_{k}$, where $I_{k}$ denotes the identity operator of $\mathcal{X}_{k}$ and $C_{k}:=$ $A_{k}^{*}\left(A_{k} V_{k}^{-1} A_{k}^{*}\right)^{\dagger} A_{k} V_{k}^{-1}$. Notice that Suzukawa (1997) showed that the previous LCA with linear constraints is the LCA of two variates $\tilde{X}_{1}:=Q_{1} X_{1}$ and $\widetilde{X}_{2}:=Q_{2} X_{2}$, where $Q_{1}$ and $Q_{2}$ are suitable orthogonal operators. In fact, this result is equivalent to the preceding one. Indeed, the LCA of $Z_{1}$ and $Z_{2}$ is the research of canonical variates $\left(\alpha_{i}, \beta_{i}\right)_{1 \leq i \leq m}\left(m \in \mathbb{N}^{*}\right)$ and canonical coefficients $\left(\rho_{i}\right)_{1 \leq i \leq m}$ satisfying:

$$
\left\{\begin{array}{l}
\widetilde{V}_{12} \widetilde{V}_{2}^{\dagger} \widetilde{V}_{21} \alpha_{i}=\rho_{i}^{2} \widetilde{V}_{1} \alpha_{i}, \quad\left\langle\alpha_{i}, \widetilde{V}_{1} \alpha_{i}\right\rangle_{\mathcal{X}_{1}=1,} \quad\left\langle\alpha_{i}, \widetilde{V}_{1} \alpha_{j}\right\rangle_{\mathcal{X}_{1}}=0 \\
\widetilde{V}_{21} \widetilde{V}_{1}^{\dagger} \widetilde{V}_{12} \beta_{i}=\rho_{i}^{2} \widetilde{V}_{2} \beta_{i}, \quad\left\langle\beta_{i}, \widetilde{V}_{2} \beta_{i}\right\rangle_{\mathcal{X}_{2}}=1, \quad\left\langle\beta_{i}, \widetilde{V}_{2} \beta_{j}\right\rangle_{\mathcal{X}_{2}}=0
\end{array} \quad \text { (for } i \neq j\right),
$$

where $\widetilde{V}_{12}=\mathbb{E}\left(Z_{2} \otimes Z_{1}\right)=\widetilde{V}_{12}^{*}$ and $\widetilde{V}_{k}=\mathbb{E}\left(Z_{k}^{\otimes^{2}}\right)(k \in\{1,2\})$. Since

$$
\begin{aligned}
& \widetilde{V}_{k}=\left(I_{k}-C_{k}\right) V_{k}\left(I_{k}-C_{k}\right)^{*}=\left(I_{k}-C_{k}\right) V_{k}=V_{k}\left(I_{k}-C_{k}\right)^{*}, \\
& \widetilde{V}_{12}=\left(I_{1}-C_{1}\right) V_{12}\left(I_{2}-C_{2}\right)^{*}
\end{aligned}
$$

and

$$
\tilde{V}_{k}^{\dagger}=\left(I_{k}-C_{k}\right)^{*} V_{k}^{-1}\left(I_{k}-C_{k}\right), \quad\left(I_{k}-C_{k}\right)^{*} V_{k}^{-1}=V_{k}^{-1}\left(I_{k}-C_{k}\right),
$$

the preceding system is equivalent to

$$
\left\{\begin{array}{lll}
\left(I_{1}-C_{1}\right) V_{12} V_{2}^{-1}\left(I_{2}-C_{2}\right) V_{21} \gamma_{i}=\rho_{i}^{2} V_{1} \gamma_{i}, & \left\langle\gamma_{i}, V_{1} \gamma_{i}\right\rangle_{\mathcal{X}_{1}}=1, & \left\langle\gamma_{i}, V_{1} \gamma_{j}\right\rangle_{\mathcal{X}_{1}}=0 \\
\left(I_{2}-C_{2}\right) V_{21} V_{1}^{-1}\left(I_{1}-C_{1}\right) V_{12} \xi_{i}=\rho_{i}^{2} V_{2} \xi_{i}, & \left\langle\xi_{i}, V_{2} \xi_{i}\right\rangle_{\mathcal{X}_{2}}=1, & \left\langle\xi_{i}, V_{2} \xi_{j}\right\rangle_{\mathcal{X}_{2}}=0
\end{array}\right.
$$

(for $i \neq j$ ) 
with $\gamma_{i}=\left(I_{1}-C_{1}\right)^{*} \alpha_{i}, \xi_{i}=\left(I_{2}-C_{2}\right)^{*} \beta_{i}$. This later system is that is shown in Suzukawa (1997) to define the LCA of $\widetilde{X}_{1}$ and $\widetilde{X}_{2}$.

Example 2.2. Relative discriminant analysis. Let $Y$ be a discrete r.v. valued into $\{1, \ldots, q\}$ and defining $q$ groups. Then put $X_{1}:=\left(\mathbf{1}_{\{Y=1\}}, \ldots, \mathbf{1}_{\{Y=q\}}\right)$; we define the relative discriminant analysis (RDA) of $Y$ and $X_{2}$ relative to $X_{3}$ as the canonical analysis of $E_{1}$ and $E_{2 \cdot 3}$. Since $E_{2 \cdot 3}=R\left(L_{X_{2 \cdot 3}}\right)$, this RDA is the discriminant analysis of $Y$ and $X_{2.3}$. This method have been introduced in the literature (see, e.g., Fujikoshi and Khatri (1990), Baccini et al. (2001)) for the case where $X_{3}$ is a covariate having the same mean in the preceding $q$ groups and admiting an invertible covariance operator.

Invariance of multivariate analyses when the related variables are transformed by linear maps have been considered in some particular forms in the literature. For instance, the problem of additional information, tackled in Fujikoshi (1892) and Suzukawa and Sato (1996) for LCA and in Fujikoshi and Khatri (1990) for covariate discriminant analysis, defined as the research of conditions for which the results of a given analysis are the same whether one considers some variables or subcomponents of them, is clearly a problem of invariance of this analysis after transformations of these variables by projectors (see Remark 2.3). Then, it is of interest to generalize the approach of the previous works by searching for conditions such that the considered analysis is invariant when the variables are transformed by linear maps which may not be projectors. This is an important goal since in multivariate analysis it often occurs that, in order to reduce dimensions, one have to work with linear transformations, and not necessarily projections, of original variables; so it may be convenient that these transformations do not affect the results of the given analysis. For the case of linear canonical analysis (LCA), this generalizing approach have been tackled by Dauxois and Nkiet (1997a) who determined conditions for having the aforementioned invariance. We will now extend this problem to the case of LRCA. For $k \in\{1,2\}$, consider an Euclidean space $\mathcal{X}_{k}^{\prime}$ with dimension $q_{k}$, a linear map $A_{k}$ from $\mathcal{X}_{k}$ to $\mathcal{X}_{k}^{\prime}$ and the r.v. $Y_{k}=A_{k} X_{k}$. It is easy to verify that, defining $W_{k 3}:=E\left(X_{3} \otimes Y_{k}\right), Y_{k \cdot 3}:=Y_{k}-W_{k 3} V_{3}^{\dagger} X_{3}$ and $W_{k \cdot 3}:=E\left(Y_{k \cdot 3}^{\otimes^{2}}\right)$, one has $W_{k 3}=A_{k} V_{k 3}$, $Y_{k \cdot 3}=A_{k} X_{k \cdot 3}$; this implies: $L_{Y_{k \cdot 3}}=L_{k \cdot 3} A_{k}^{*}$. The LRCA of $X_{1}$ and $X_{2}$ relative to $X_{3}$ is the triple given in equation (2.6), and similarly we consider the triple

$$
\left\{\left(\gamma_{i}\right)_{1 \leq i \leq s},\left(\beta_{1 \cdot 3}^{(i)}\right)_{1 \leq i \leq q_{1}},\left(\beta_{2 \cdot 3}^{(i)}\right)_{1 \leq i \leq q_{2}}\right\}
$$

which characterizes the LRCA of $Y_{1}$ and $Y_{2}$ relative to $X_{3}$. The canonical variates corresponding to the preceding LRCA are

and

$$
f_{k \cdot 3}^{(i)}=L_{k \cdot 3}\left(V_{k \cdot 3}^{\dagger}\right)^{1 / 2} \alpha_{k \cdot 3}^{(i)}, \quad 1 \leq i \leq p_{k}
$$

$$
g_{k \cdot 3}^{(i)}=L_{Y_{k \cdot 3}}\left(W_{k \cdot 3}^{\dagger}\right)^{1 / 2} \beta_{k \cdot 3}^{(i)}, \quad 1 \leq i \leq q_{k}
$$

DEFinition 2.2. The LRCA of $X_{1}$ and $X_{2}$ is invariant for the pair $\left(A_{1}, A_{2}\right)$ if the following conditions are satisfied:

(i) $r=s$ and $\rho_{i}=\gamma_{i}(i=1, \ldots, r)$;

(ii) for all $(k, i) \in\{1,2\} \times\{1, \ldots, r\}, f_{k \cdot 3}^{(i)}=g_{k \cdot 3}^{(i)}$. 
Now, we can seek a necessary and sufficient condition for which this invariance property holds. Notice that, since $Y_{k \cdot 3}=A_{k} X_{k \cdot 3}(k=1,2)$, the invariance introduced in the previous definition means the invariance of the LCA of $X_{1.3}$ and $X_{2.3}$ for the pair $\left(A_{1}, A_{2}\right)$. Then, by applying Proposition 4.2 of Dauxois and Nkiet $(1997 a)$, we obtain:

Proposition 2.2. The LRCA of $X_{1}$ and $X_{2}$ is invariant for the pair $\left(A_{1}, A_{2}\right)$ if, and only if one has:

$$
V_{12 \cdot 3}=V_{1 \cdot 3} A_{1}^{*}\left(A_{1} V_{1 \cdot 3} A_{1}^{*}\right)^{\dagger} A_{1} V_{12 \cdot 3} \quad \text { and } \quad V_{21 \cdot 3}=V_{2 \cdot 3} A_{2}^{*}\left(A_{2} V_{2 \cdot 3} A_{2}^{*}\right)^{\dagger} A_{2} V_{21 \cdot 3}
$$

Remark 2.3. The previous notion of invariance for LRCA is related to the problem of additional information in canonical analysis which interested some authors. For example, Siotani (1957) studied the effect of adding variates on the canonical coefficients and Fujikoshi (1982) determined conditions for which LCA remains unchanged when subcomponents of the involved variates are omitted. When, for $k \in\{1,2\}$, we have the decomposition in direct sum $\mathcal{X}_{k}=\mathcal{X}_{k}^{(1)} \oplus \mathcal{X}_{k}^{(2)}$, an analogous problem of additional information can be formulated for LRCA. Consider $\pi_{k 1}$ (resp. $\pi_{k 2}$ ) the projection operator on $\mathcal{X}_{k}^{(1)}$ (resp. $\mathcal{X}_{k}^{(2)}$ ) along $\mathcal{X}_{k}^{(2)}$ (resp. $\mathcal{X}_{k}^{(1)}$ ) and put:

$$
X_{k j}=\pi_{k j} X_{k} \quad(j=1,2) .
$$

We say that the pair $\left(X_{12}, X_{22}\right)$ does not provide additional information on the LRCA of $X_{1}$ and $X_{2}$ relative to $X_{3}$ if this later LRCA is invariant for the pair $\left(\pi_{11}, \pi_{21}\right)$. A necessary and sufficient condition for this invariance is obtained by applying Proposition 2.2 to the pair $\left(\pi_{11}, \pi_{21}\right)$; now, by taking $X_{3}=0$ we obtain the condition of Fujikoshi (1982).

\section{Asymptotic study of LRCA}

The asymptotic theory for classical LCA is well known; the earlier works on this subject focused on the asymptotic joint distribution of the sample canonical correlation coefficients under normality (see Hsu (1941)) or nonnormality (see Muirhead and Waternaux (1980)). Later, asymptotic distributions both for these coefficients and for sample canonical vectors and/or projections were derived under normality and when the population canonical correlation coefficients are distinct (see Anderson (1999) and references inside) or under nonnormality and in case the preceding coefficients have multiplicities (see Arconte (1980), Pousse (1992), Larrère (1994) and Fine (2000)). In fact, asymptotic study for LCA or others multivariate statistical analyses reduces to determining consistency and asymptotic distributions for eigenvalues, eigenvectors and eigenprojections of an operator which is known to be consistent and for which an asymptotic distribution is known. That is not technically difficult nowadays since one can apply results of DossouGbete and Pousse (1991) for a selfadjoint random operator, or those of Eaton and Tyler (1994) when one focuses on singular values of a random matrix which may be not symmetric. Finally, making an asymptotic study for a statistical multivariate analysis mainly consists in studying the consistency and in deriving an asymptotic distribution for the related operator.

In this section, we focus on asymptotics for LRCA. Although it is a particular LCA, the results of asymptotic study for this later analysis cannot been applied to 
it. Indeed, these results hold when an i.i.d. sample of the related random variables is avalaible; but, as we will see below, we don't have any sample of $\left(X_{1 \cdot 3}, X_{2 \cdot 3}\right)$. These r.v. are unobservable ones because their definitions (see equation (2.4)) involve covariance operators which are unknown in practice. Then, consistency and asymptotic distribution for the sample operator related to LRCA are not straightforward and there is an interest to determine them.

We suppose that, for all $(k, m) \in\{1,2\} \times\{1,2,3\}$, we have:

(A1) $\mathbb{E}\left(\left\|X_{m}\right\|_{\mathcal{X}_{m}}^{4}\right)<+\infty$;

(A2) $V_{m}$ is invertible;

(A3) $E_{k} \cap E_{3}=\{0\}$;

(A4) $V_{k \cdot 3}=I_{k}$

where $I_{k}$ denotes the identity of $\mathcal{X}_{k}$.

Remark 3.1. From Lemma 2.2 we know that the assumptions (A2) and (A3) imply thas $V_{k \cdot 3}$ is invertible. Then Assumption (A4) does not restrict the generality; indeed one can always reduce to that situation by considering $Y_{k}=V_{k \cdot 3}^{-1 / 2} X_{k}$ instead of $X_{k}$ $(k=1,2)$, and since $V_{k \cdot 3}^{-1 / 2}$ is invertible the transformation $X_{k} \mapsto V_{k \cdot 3}^{-1 / 2} X_{k}$ yields invariance of the LRCA.

Let $\left(X_{1}^{(i)}, X_{2}^{(i)}, X_{3}^{(i)}\right)_{1 \leq i \leq n}$ an i.i.d. sample of the triple $Z=\left(X_{1}, X_{2}, X_{3}\right)$; for $(m, k, j) \in\{1,2,3\} \times\{1,2\}^{2}$, we consider:

$$
\begin{aligned}
& \bar{X}_{m}^{(n)}=\frac{1}{n} \sum_{i=1}^{n} X_{m}^{(i)}, \\
& V_{m}^{(n)}=\frac{1}{n} \sum_{i=1}^{n}\left(X_{m}^{(i)}-\bar{X}_{m}^{(n)}\right)^{\otimes^{2}}, \\
& V_{k j}^{(n)}=\frac{1}{n} \sum_{i=1}^{n}\left(X_{j}^{(i)}-\bar{X}_{j}^{(n)}\right) \otimes\left(X_{k}^{(i)}-\bar{X}_{k}^{(n)}\right)=V_{j k}^{(n)^{*}} \quad \text { for } \quad k \neq j .
\end{aligned}
$$

Notice that $V_{m}^{(n)}=\frac{1}{n} \sum_{i=1}^{n} X_{m}^{(i)^{\otimes^{2}}}-\bar{X}_{m}^{(n)^{\otimes^{2}}}$ and $V_{k j}^{(n)}=\frac{1}{n} \sum_{i=1}^{n} X_{j}^{(i)} \otimes X_{k}^{(i)}-\bar{X}_{j}^{(n)} \otimes \bar{X}_{k}^{(n)}$; then by the strong law of large numbers $V_{m}^{(n)}$ and $V_{k j}^{(n)}$ almost surely uniformly converge to $V_{m}$ and $V_{k j}$ respectively, as $n \rightarrow+\infty$. This shows that for large values of $n, V_{3}^{(n)}$ is invertible (a.s.); thus we can define

$$
\begin{aligned}
& V_{k \cdot 3}^{(n)}:=V_{k}^{(n)}-V_{k 3}^{(n)} V_{3}^{(n)^{-1}} V_{3 k}^{(n)}, \quad \text { and } \\
& V_{12 \cdot 3}^{(n)}:=V_{12}^{(n)}-V_{13}^{(n)} V_{3}^{(n)^{-1}} V_{32}^{(n)}=V_{21 \cdot 3}^{(n)^{*}} .
\end{aligned}
$$

We also have the almost sure uniform convergence of $V_{k \cdot 3}^{(n)}$ to $I_{k}$, as $n \rightarrow+\infty$. Then, for large $n, V_{k \cdot 3}^{(n)}$ also is invertible (a.s.) and we put:

$$
T_{1 \cdot 3}^{(n)}=V_{1 \cdot 3}^{(n)^{-1 / 2}} V_{12 \cdot 3}^{(n)} V_{2 \cdot 3}^{(n)^{-1}} V_{21 \cdot 3}^{(n)} V_{1 \cdot 3}^{(n)^{-1 / 2}}
$$

We take the spectral analysis of $T_{1 \cdot 3}^{(n)}$ as an estimator of the LRCA of $X_{1}$ and $X_{2}$ relative to $X_{3}$ and our goal is to study the asymptotic properties of this estimator and its eigenelements. 
Let $\lambda^{\prime}:=\left(\lambda_{j}^{\prime}\right)_{1 \leq j \leq s}\left(\right.$ resp. $\left.\lambda:=\left(\lambda_{i}\right)_{1 \leq i \leq p_{1}}\right)$ be the strictly decreasing (resp. complete nonincreasing) sequence of the eigenvalues of $T_{1 \cdot 3}$, we denote by $m_{j}$ the multiplicity of $\lambda_{j}^{\prime}$. It is clear that $\lambda_{i}=\rho_{i}^{2}$ with $\rho_{i}=0$ if $i>r$ and that, putting $\nu_{j}=1+\sum_{l=0}^{j-1} m_{l}$ (with $m_{0}=0$ ), one has $\lambda_{j}^{\prime}=\lambda_{\nu_{j}}$. Then we consider the orthogonal projection operator $P_{j}$ onto the eigenspace of $T_{1 \cdot 3}$ associated to $\lambda_{j}^{\prime}$, that is $P_{j}:=\sum_{i=\nu_{j}}^{\nu_{j}+m_{j}-1} \alpha_{1 \cdot 3}^{(i)^{\otimes^{2}}}$. Moreover, letting $\left(\lambda_{i}^{(n)}\right)_{1 \leq i \leq p_{1}}$ be the complete sequence of eigenvalues of $T_{1 \cdot 3}^{(n)}$, we consider an orthonormal basis $\left(\alpha_{1 \cdot 3, n}^{(i)}\right)_{1 \leq i \leq p_{1}}$ of associated eigenvectors such that $\alpha_{1 \cdot 3, n}^{(i)}$ is associated to $\lambda_{i}^{(n)}$, and we put $P_{j}^{(n)}:=\sum_{i=\nu_{j}}^{\nu_{j}+m_{j}-1} \alpha_{1 \cdot 3, n}^{(i)^{\otimes^{2}}}$.

\subsection{Almost sure convergence}

As already noticed, the empirical covariance operators defined in equation (3.1) and equation (3.2) almost surely uniformly converge to the corresponding covariance operators. Thus, $V_{1 \cdot 3}^{(n)}$ (resp. $V_{2 \cdot 3}^{(n)} ; V_{12 \cdot 3}^{(n)}$ ) almost surely uniformly converges to $I_{1}$ (resp. $I_{2} ; V_{12.3}$ ). Consequently, $T_{1.3}^{(n)}$ converges almost surely uniformly to $T_{1.3}$. A direct application of Proposition 3 of Dossou-Gbete and Pousse (1991) gives the following almost sure convergence properties of the eigenelements of $T_{1 \cdot 3}^{(n)}$.

Proposition 3.1. (i) For any $j \in\{1, \ldots, s\}$ and any $i \in\left\{\nu_{j}, \ldots, \nu_{j}+m_{j}-1\right\}$, $\left(\lambda_{i}^{(n)}\right)_{n \in \mathbb{N}^{*}}$ converges almost surely to $\lambda_{j}^{\prime}$.

(ii) For any $j \in\{1, \ldots, s\},\left(P_{j}^{(n)}\right)_{n \in \mathbb{N}^{*}}$ converges almost surely uniformly to $P_{j}$.

(iii) If $m_{j}=1$, then $\left(\alpha_{1 \cdot 3, n}^{\left(\nu_{j}\right)}\right)_{n \in \mathbb{N}^{*}}$ converges almost surely to $\alpha_{1 \cdot 3}^{\left(\boldsymbol{\nu}_{j}\right)}$.

\subsection{Convergence in distribution}

Here, we will derive the asymptotic distribution of $\sqrt{n}\left(T_{1 \cdot 3}^{(n)}-T_{1 \cdot 3}\right)$ and, consequently, those of the eigenelements of this operator.

We identify $\mathcal{X}_{1} \times \mathcal{X}_{2} \times \mathcal{X}_{3}$ with the direct orthogonal sum $\mathcal{X}=\mathcal{X}_{1} \oplus \mathcal{X}_{2} \oplus \mathcal{X}_{3}$, the aforementioned orthogonality being related to the inner product of $\mathcal{X}$ defined by $\langle x, y\rangle_{\mathcal{X}}=\sum_{m=1}^{3}\left\langle x_{m}, y_{m}\right\rangle_{\mathcal{X}_{m}}$ for all $x:=\sum_{m=1}^{3} x_{m} \in \mathcal{X}$ and all $y:=\sum_{m=1}^{3} y_{m} \in \mathcal{X}$. Then we can write $Z=\sum_{m=1}^{3} X_{m}$ and put $Z_{i}=\sum_{m=1}^{3} X_{m}^{(i)}(i=1, \ldots, n)$. In the same way, the space $\mathcal{L}\left(\mathcal{X}_{1} \times \mathcal{X}_{2} \times \mathcal{X}_{3}\right)$ of linear maps from $\mathcal{X}_{1} \times \mathcal{X}_{2} \times \mathcal{X}_{3}$ into itself will be identified to the orthogonal sum $M=\bigoplus_{1 \leq m, j \leq 3} \mathcal{L}\left(\mathcal{X}_{m}, \mathcal{X}_{j}\right)$, where for any pair $(F, G)$ of Euclidean spaces, we denote by $\mathcal{L}(F, G)$ the space of linear maps from $F$ into $G$ (when $F=G$, we will write $\mathcal{L}(F)$ ). Hence we can write $V=\mathbb{E}\left(Z^{\otimes^{2}}\right)=\sum_{1 \leq m, j \leq 3} V_{j m}$ (with $V_{j j}:=V_{j}$ ). Let us consider:

$$
\begin{aligned}
& \bar{Z}^{(n)}=\frac{1}{n} \sum_{i=1}^{n} Z_{i}=\sum_{m=1}^{3} \bar{X}_{m}^{(n)} \\
& V_{n}=\frac{1}{n} \sum_{i=1}^{n}\left(Z_{i}-\bar{Z}^{(n)}\right)^{\otimes^{2}}=\sum_{1 \leq j, m \leq 3} V_{j m}^{(n)} \quad\left(\text { with } \quad V_{j j}^{(n)}:=V_{j}^{(n)}\right)
\end{aligned}
$$

and

$$
H_{n}=\sqrt{n}\left(V_{n}-V\right)=\sum_{1 \leq j, m \leq 3} \sqrt{n}\left(V_{j m}^{(n)}-V_{j m}\right)
$$


For $(r, s) \in\{1,2,3\}^{2}$, we denote by $p_{r s}$ the orthogonal projection operator from $M=\bigoplus_{1 \leq m, j \leq 3} \mathcal{L}\left(\mathcal{X}_{m}, \mathcal{X}_{j}\right)$ to $\mathcal{L}\left(\mathcal{X}_{s}, \mathcal{X}_{r}\right)$, that is the operator such that for all $A=$ $\sum_{1 \leq i, j \leq 3} \bar{A}_{i j} \in M$, one has $p_{r s}(A)=A_{r s}$. First, we will prove three useful lemmas.

LEMMA 3.1. For $k \in\{1,2\}$, one has $\sqrt{n}\left(V_{k \cdot 3}^{(n)}-I_{k}\right)=a_{k \cdot 3}^{(n)}\left(H_{n}\right)$, where $H_{n}$ is defined in equation (3.5) and $\left(a_{k \cdot 3}^{(n)}\right)_{n \in \mathbb{N}^{*}}$ is a sequence of random operators from $M$ to $\mathcal{L}\left(\mathcal{X}_{k}\right)$ which converges almost surely uniformly to the operator $a_{k \cdot 3}$ of $\mathcal{L}\left(M, \mathcal{L}\left(\mathcal{X}_{k}\right)\right)$ defined by:

$$
a_{k \cdot 3}(A)=p_{k k}(A)-p_{k 3}(A) V_{3}^{-1} V_{3 k}+V_{k 3} V_{3}^{-1} p_{33}(A) V_{3}^{-1} V_{3 k}-V_{k 3} V_{3}^{-1} p_{3 k}(A)
$$

ProOF. Using equation (3.3) and $I_{k}=V_{k \cdot 3}=V_{k}-V_{k 3} V_{3}^{-1} V_{3 k}$, we have:

$$
\begin{aligned}
V_{k \cdot 3}^{(n)}-I_{k}= & V_{k}^{(n)}-V_{k 3}^{(n)} V_{3}^{(n)^{-1}} V_{3 k}^{(n)}-I_{k} \\
= & V_{k}^{(n)}-V_{k 3}^{(n)} V_{3}^{(n)^{-1}} V_{3 k}^{(n)}-V_{k}+V_{k 3} V_{3}^{-1} V_{3 k} \\
= & \left(V_{k}^{(n)}-V_{k}\right)-\left(V_{k 3}^{(n)}-V_{k 3}\right) V_{3}^{(n)^{-1}} V_{3 k}^{(n)} \\
& +V_{k 3} V_{3}^{(n)^{-1}}\left(V_{3}^{(n)}-V_{3}\right) V_{3}^{-1} V_{3 k}^{(n)}-V_{k 3} V_{3}^{-1}\left(V_{3 k}^{(n)}-V_{3 k}\right) .
\end{aligned}
$$

Hence $\sqrt{n}\left(V_{k \cdot 3}^{(n)}-I_{k}\right)=a_{k \cdot 3}^{(n)}\left(H_{n}\right)$, where $a_{k \cdot 3}^{(n)}$ is the random operator of $\mathcal{L}\left(M, \mathcal{L}\left(\mathcal{X}_{k}\right)\right)$ defined by:

$$
a_{k \cdot 3}^{(n)}(A)=p_{k k}(A)-p_{k 3}(A) V_{3}^{(n)^{-1}} V_{3 k}^{(n)}+V_{k 3} V_{3}^{(n)^{-1}} p_{33}(A) V_{3}^{-1} V_{3 k}^{(n)}-V_{k 3} V_{3}^{-1} p_{3 k}(A)
$$

Then, the almost sure uniform convergence $\left(a_{k \cdot 3}^{(n)}\right)_{n \in \mathbb{N}^{*}}$ to $a_{k \cdot 3}$ is obviously deduced from that of $V_{3}^{(n)}$ (resp. $V_{3 k}^{(n)}$ ) to $V_{3}$ (resp. $V_{3 k}$ ).

LEMMA 3.2. One has $\sqrt{n}\left(V_{12 \cdot 3}^{(n)}-V_{12 \cdot 3}\right)=a_{12 \cdot 3}^{(n)}\left(H_{n}\right)$, where $\left(a_{12 \cdot 3}^{(n)}\right)_{n \in \mathbb{N}^{*}}$ is a sequence of random operators from $M$ to $\mathcal{L}\left(\mathcal{X}_{2}, \mathcal{X}_{1}\right)$ which converges almost surely uniformly to the operator $a_{12.3}$ of $\mathcal{L}\left(M, \mathcal{L}\left(\mathcal{X}_{2}, \mathcal{X}_{1}\right)\right)$ defined by:

$$
a_{12 \cdot 3}(A)=p_{12}(A)-p_{13}(A) V_{3}^{-1} V_{32}+V_{13} V_{3}^{-1} p_{33}(A) V_{3}^{-1} V_{32}-V_{13} V_{3}^{-1} p_{32}(A) .
$$

Proof. Using equations (2.5) and (3.4), we can write:

$$
\begin{aligned}
V_{12 \cdot 3}^{(n)}-V_{12 \cdot 3}= & V_{12}^{(n)}-V_{12}-V_{13}^{(n)} V_{3}^{(n)^{-1}} V_{32}^{(n)}+V_{13} V_{3}^{-1} V_{32} \\
= & \left(V_{12}^{(n)}-V_{12}\right)-\left(V_{13}^{(n)}-V_{13}\right) V_{3}^{(n)^{-1}} V_{32}^{(n)} \\
& +V_{13} V_{3}^{(n)^{-1}}\left(V_{3}^{(n)}-V_{3}\right) V_{3}^{-1} V_{32}^{(n)}-V_{13} V_{3}^{-1}\left(V_{32}^{(n)}-V_{32}\right)
\end{aligned}
$$

Thus we have $\sqrt{n}\left(V_{12 \cdot 3}^{(n)}-V_{12 \cdot 3}\right)=a_{12 \cdot 3}^{(n)}\left(H_{n}\right)$, where $a_{12 \cdot 3}^{(n)} \in \mathcal{L}\left(M, \mathcal{L}\left(\mathcal{X}_{2}, \mathcal{X}_{1}\right)\right)$ is defined by:

$$
a_{12 \cdot 3}^{(n)}(A)=p_{12}(A)-p_{13}(A) V_{3}^{(n)^{-1}} V_{32}^{(n)}+V_{13} V_{3}^{(n)^{-1}} p_{33}(A) V_{3}^{-1} V_{32}^{(n)}-V_{13} V_{3}^{-1} p_{32}(A) .
$$


Then, the almost sure uniform convergence $\left(a_{k \cdot 3}^{(n)}\right)_{n \in \mathbb{N}^{*}}$ to $a_{k \cdot 3}$ is obviously deduced from that of $V_{3}^{(n)}$ (resp. $V_{32}^{(n)}$ ) to $V_{3}$ (resp. $V_{32}$ ).

Similarly, we also have:

LEMmA 3.3. One has $\sqrt{n}\left(V_{21 \cdot 3}^{(n)}-V_{21 \cdot 3}\right)=a_{21 \cdot 3}^{(n)}\left(H_{n}\right)$, where $\left(a_{21 \cdot 3}^{(n)}\right)_{n \in \mathbb{N}^{*}}$ is a sequence of random operators from $M$ to $\mathcal{L}\left(\mathcal{X}_{1}, \mathcal{X}_{2}\right)$ which converges almost surely uniformly to the operator $a_{21 \cdot 3}$ of $\mathcal{L}\left(M, \mathcal{L}\left(\mathcal{X}_{1}, \mathcal{X}_{2}\right)\right)$ defined by:

$$
a_{21 \cdot 3}(A)=p_{21}(A)-p_{23}(A) V_{3}^{-1} V_{31}+V_{23} V_{3}^{-1} p_{33}(A) V_{3}^{-1} V_{31}-V_{23} V_{3}^{-1} p_{31}(A) \text {. }
$$

These lemmas permit us to obtain the asymptotic distribution of $\sqrt{n}\left(T_{1 \cdot 3}^{(n)}-T_{1 \cdot 3}\right)$. In what follows, we consider the operator:

$$
\pi: A \in \mathcal{L}\left(\mathcal{X}_{1}\right) \mapsto \frac{1}{2}\left(A+A^{*}\right) \in \mathcal{L}\left(\mathcal{X}_{1}\right)
$$

that is the orthogonal projector onto the subspace of the selfadjoint operators in $\mathcal{L}\left(X_{1}\right)$ and $\widetilde{\otimes}$ denotes the tensor product of operators, associated with the Hilbert-Schmidt inner product: $\langle T, S\rangle_{2}=\operatorname{tr}\left(T S^{*}\right)$.

Proposition 3.2. $\sqrt{n}\left(T_{1 \cdot 3}^{(n)}-T_{1 \cdot 3}\right)$ converges in distribution, as $n \rightarrow+\infty$, to a r.v. $U$ having a centered normal distribution in $\mathcal{L}\left(\mathcal{X}_{1}\right)$ with covariance operator given by:

$$
\Gamma=\mathbb{E}\left[\left(\pi\left(-V_{12 \cdot 3} V_{21 \cdot 3} X_{1 \cdot 3}^{\otimes^{2}}-V_{12 \cdot 3} X_{2 \cdot 3}^{\otimes^{2}} V_{21 \cdot 3}+2 V_{12 \cdot 3}\left(X_{1 \cdot 3} \otimes X_{2 \cdot 3}\right)\right)\right)^{\tilde{\otimes}^{2}}\right] .
$$

Proof. We have:

$$
\begin{aligned}
\sqrt{n}\left(T_{1 \cdot 3}^{(n)}-T_{1 \cdot 3}\right)= & \sqrt{n}\left(V_{1 \cdot 3}^{(n)-1 / 2}-I_{1}\right) V_{12 \cdot 3}^{(n)} V_{2 \cdot 3}^{(n)^{-1}} V_{21 \cdot 3}^{(n)} V_{1 \cdot 3}^{(n)-1 / 2} \\
& +\left[\sqrt{n}\left(V_{12 \cdot 3}^{(n)}-V_{12 \cdot 3}\right)\right] V_{2 \cdot 3}^{(n)^{-1}} V_{21 \cdot 3}^{(n)} V_{1 \cdot 3}^{(n)-1 / 2} \\
& -V_{12 \cdot 3} V_{2 \cdot 3}^{(n)^{-1}}\left[\sqrt{n}\left(V_{2 \cdot 3}^{(n)}-I_{2}\right)\right] V_{21 \cdot 3}^{(n)} V_{1 \cdot 3}^{(n)-1 / 2} \\
& +V_{12 \cdot 3}\left[\sqrt{n}\left(V_{21 \cdot 3}^{(n)}-V_{21 \cdot 3}\right)\right] V_{1 \cdot 3}^{(n)-1 / 2} \\
& +V_{12 \cdot 3} V_{21 \cdot 3}\left[\sqrt{n}\left(V_{1 \cdot 3}^{(n)-1 / 2}-I_{1}\right)\right]
\end{aligned}
$$

For any invertible selfadjoint nonegative operator $T$, one has

$$
T^{-1 / 2}-I=-T^{-1 / 2}(T-I)\left(T^{-1 / 2}+I\right)^{-1}
$$

where $I$ denotes the identity. Then applying this equality with $T=V_{1 \cdot 3}^{(n)}$ and using the three previous lemmas, we obtain:

$$
\begin{aligned}
\sqrt{n}\left(T_{1 \cdot 3}^{(n)}-T_{1 \cdot 3}\right)= & -V_{1 \cdot 3}^{(n)^{-1}} a_{1 \cdot 3}^{(n)}\left(H_{n}\right)\left(V_{1 \cdot 3}^{(n)^{-1 / 2}}+I_{1}\right)^{-1} V_{12 \cdot 3}^{(n)} V_{2 \cdot 3}^{(n)^{-1}} V_{21 \cdot 3}^{(n)} V_{1 \cdot 3}^{(n)^{-1 / 2}} \\
& +a_{12 \cdot 3}^{(n)}\left(H_{n}\right) V_{2 \cdot 3}^{(n)^{-1}} V_{21 \cdot 3}^{(n)} V_{1 \cdot 3}^{(n)^{-1 / 2}}-V_{12 \cdot 3} V_{2 \cdot 3}^{(n)^{-1}} a_{2 \cdot 3}^{(n)}\left(H_{n}\right) V_{21 \cdot 3}^{(n)} V_{1 \cdot 3}^{(n)^{-1 / 2}} \\
& +V_{12 \cdot 3} a_{21 \cdot 3}^{(n)}\left(H_{n}\right) V_{1 \cdot 3}^{(n)-1 / 2} \\
& -V_{12 \cdot 3} V_{21 \cdot 3} V_{1 \cdot 3}^{(n)^{-1}} a_{1 \cdot 3}^{(n)}\left(H_{n}\right)\left(V_{1 \cdot 3}^{(n)^{-1 / 2}}+I_{1}\right)^{-1} \\
= & \varphi_{n}\left(H_{n}\right) .
\end{aligned}
$$


From the almost sure uniform convergence of $V_{12 \cdot 3}^{(n)}, V_{1 \cdot 3}^{(n)}$ and $V_{2 \cdot 3}^{(n)}$, as $n \rightarrow+\infty$, to $V_{12.3}, I_{1}$ and $I_{2}$ respectively, we deduce that $\left(\varphi_{n}\right)_{n \in \mathbb{N}^{*}}$ almost surely uniformly converges to the operator $\varphi \in \mathcal{L}\left(M, \mathcal{L}\left(\mathcal{X}_{1}\right)\right)$ defined by:

$$
\begin{aligned}
\varphi(A)= & -\frac{1}{2}\left[a_{1 \cdot 3}(A) V_{12 \cdot 3} V_{21 \cdot 3}+V_{12 \cdot 3} V_{21 \cdot 3} a_{1 \cdot 3}(A)\right]+a_{12 \cdot 3}(A) V_{21 \cdot 3} \\
& +V_{12 \cdot 3} a_{21 \cdot 3}(A)-V_{12 \cdot 3} a_{2 \cdot 3}(A) V_{21 \cdot 3} .
\end{aligned}
$$

Further, we have:

$$
H_{n}=\sqrt{n}\left[\frac{1}{n} \sum_{i=1}^{n} Z_{i}^{\otimes^{2}}-V\right]-\bar{Z}^{(n)^{\otimes^{2}}} .
$$

By the central limit theorem $\sqrt{n} \bar{Z}^{(n)}$ converges in distribution to a r.v. having a centered normal distribution in $\mathcal{X}$ with covariance operator $\mathbb{E}\left(Z^{\otimes^{2}}\right)$, this implies that $\bar{Z}^{(n)}$ converges in probability to 0 in $\mathcal{X}$, as $n \rightarrow+\infty$. Hence $H_{n}$ has the same asymptotic distribution than $\sqrt{n}\left[\frac{1}{n} \sum_{i=1}^{n} Z_{i}^{\otimes^{2}}-V\right]$; thus, by the central limit theorem, $H_{n}$ converges in distribution, as $n \rightarrow+\infty$, to a r.v. $H$ having a centered normal distribution in $M$, with covariance operator defined by $\mathbb{E}\left[\left(Z^{\otimes^{2^{c}}}\right)^{\tilde{\otimes}^{2}}\right]$, where $Z^{\otimes^{2^{c}}}:=Z^{\otimes^{2}}-\mathbb{E}\left(Z^{\otimes^{2}}\right)$. Moreover, we have

$$
\left\|\varphi_{n}\left(H_{n}\right)-\varphi\left(H_{n}\right)\right\|_{\mathcal{L}\left(\mathcal{X}_{1}\right)} \leq\left\|\varphi_{n}-\varphi\right\|_{\infty}\left\|H_{n}\right\|_{M},
$$

where $\|\cdot\|_{\infty}$ is the uniform convergence norm. Since $\left\|H_{n}\right\|_{M}$ (resp. $\left\|\varphi_{n}-\varphi\right\|_{\infty}$ ) converges in distribution (resp. in probability), as $n \rightarrow+\infty$, to $\|H\|_{M}$ (resp. 0), the previous inequality shows that $\varphi_{n}\left(H_{n}\right)-\varphi\left(H_{n}\right)$ converges in probability, in $\mathcal{L}\left(\mathcal{X}_{1}\right)$, to 0 as $n \rightarrow$ $+\infty$. Hence $\varphi_{n}\left(H_{n}\right)$ has the same asymptotic distribution than $\varphi\left(H_{n}\right)$, that is the distribution of $\varphi(H)$ because $\varphi$ is linear. This means that $\sqrt{n}\left(T_{1.3}^{(n)}-T_{1.3}\right)$ converges to the same centered normal distribution than $U=\varphi(H)$; the related covariance operator is:

$$
\Gamma=\mathbb{E}\left[(\varphi(H))^{\tilde{\otimes}^{2}}\right]=\varphi \mathbb{E}\left[H^{\tilde{\otimes}^{2}}\right] \varphi^{*}=\varphi \mathbb{E}\left[\left(Z^{\otimes^{2^{c}}}\right)^{\dot{\otimes}^{2}}\right] \varphi^{*}=\mathbb{E}\left[\left(\varphi\left(Z^{\otimes^{2^{c}}}\right)\right)^{\tilde{\otimes}^{2}}\right] .
$$

It remains to give an explicit expression of $\Gamma$. We have:

$$
\begin{aligned}
a_{1 \cdot 3}\left(Z^{\otimes^{2^{c}}}\right)= & X_{1}^{\otimes^{2}}-V_{1}-\left(X_{3} \otimes X_{1}-V_{13}\right) V_{3}^{-1} V_{31} \\
& +V_{13} V_{3}^{-1}\left(X_{3}^{\otimes^{2}}-V_{3}\right) V_{3}^{-1} V_{31}-V_{13} V_{3}^{-1}\left(X_{1} \otimes X_{3}-V_{31}\right) \\
= & X_{1}^{\otimes^{2}}-\left(V_{13} V_{3}^{-1} X_{3}\right) \otimes X_{1}-X_{1} \otimes\left(V_{13} V_{3}^{-1} X_{3}\right) \\
& +\left(V_{13} V_{3}^{-1} X_{3}\right)^{\otimes^{2}}-V_{1}+V_{13} V_{3}^{-1} V_{31} \\
= & X_{1 \cdot 3}^{\otimes^{2}}-I_{1}, \\
a_{12 \cdot 3}\left(Z^{\otimes^{2^{c}}}=\right. & X_{2} \otimes X_{1}-V_{12}-\left(X_{3} \otimes X_{1}-V_{13}\right) V_{3}^{-1} V_{32} \\
& +V_{13} V_{3}^{-1}\left(X_{3}^{\otimes^{2}}-V_{3}\right) V_{3}^{-1} V_{32}-V_{13} V_{3}^{-1}\left(X_{2} \otimes X_{3}-V_{32}\right) \\
= & X_{2} \otimes X_{1}-\left(V_{23} V_{3}^{-1} X_{3}\right) \otimes X_{1}-X_{2} \otimes\left(V_{13} V_{3}^{-1} X_{3}\right) \\
& +\left(V_{23} V_{3}^{-1} X_{3}\right) \otimes\left(V_{13} V_{3}^{-1} X_{3}\right)-V_{12}+V_{13} V_{3}^{-1} V_{32} \\
= & X_{2 \cdot 3} \otimes X_{1 \cdot 3}-V_{12.3} ;
\end{aligned}
$$


similarly

and

$$
a_{21 \cdot 3}\left(Z^{\otimes^{2^{c}}}\right)=X_{1 \cdot 3} \otimes X_{2 \cdot 3}-V_{21 \cdot 3}
$$

$$
a_{2 \cdot 3}\left(Z^{\otimes^{c}}\right)=X_{2 \cdot 3}^{\otimes^{2}}-I_{2}
$$

Hence

$$
\begin{aligned}
\varphi\left(Z^{\otimes^{2^{c}}}\right)= & -\frac{1}{2}\left[\left(X_{1 \cdot 3}^{\otimes^{2}}-I_{1}\right) V_{12 \cdot 3} V_{21 \cdot 3}+V_{12 \cdot 3} V_{21 \cdot 3}\left(X_{1 \cdot 3}^{\otimes^{2}}-I_{1}\right)\right] \\
& +\left(X_{2 \cdot 3} \otimes X_{1 \cdot 3}-V_{12 \cdot 3}\right) V_{21 \cdot 3}+V_{12 \cdot 3}\left(X_{1 \cdot 3} \otimes X_{2 \cdot 3}-V_{21 \cdot 3}\right) \\
& -V_{12 \cdot 3}\left(X_{2 \cdot 3}^{\otimes^{2}}-I_{2}\right) V_{21 \cdot 3} \\
= & \pi\left(-V_{12 \cdot 3} V_{21 \cdot 3} X_{1 \cdot 3}^{\otimes^{2}}-V_{12 \cdot 3} X_{2 \cdot 3}^{\otimes^{2}} V_{21 \cdot 3}+2 V_{12 \cdot 3}\left(X_{1 \cdot 3} \otimes X_{2 \cdot 3}\right)\right)
\end{aligned}
$$

this completes the proof.

This proposition permits us to obtain the asymptotic distributions of the eigenelements. For $j \in\{1, \ldots, s\}$, considering the operators

$$
\begin{aligned}
& S_{j}=\sum_{1 \leq l \leq s, l \neq j} \frac{1}{\lambda_{j}^{\prime}-\lambda_{l}^{\prime}} P_{l} \\
& \Psi_{j}: T \in \mathcal{L}\left(\mathcal{X}_{1}\right) \mapsto P_{j} T S_{j}+S_{j} T P_{j} \in \mathcal{L}\left(\mathcal{X}_{1}\right) \\
& \Psi_{j}^{\prime}: T \in \mathcal{L}\left(\mathcal{X}_{1}\right) \mapsto P_{j} T P_{j} \in \mathcal{L}\left(\mathcal{X}_{1}\right) \\
& \Theta_{j}: T \in \mathcal{L}\left(\mathcal{X}_{1}\right) \mapsto S_{j} T \alpha_{1 \cdot 3}^{\left(\nu_{j}\right)} \in \mathcal{L}\left(\mathcal{X}_{1}\right),
\end{aligned}
$$

denoting by $\Delta$ the continuous map which associates to $T \in \mathcal{L}\left(\mathcal{X}_{1}\right)$ its complete nonincreasing sequence of eigenvalues, and putting $\rho_{i}^{(n)}:=\sqrt{\lambda_{i}^{(n)}}, \rho_{j}^{\prime}:=\sqrt{\lambda_{j}^{\prime}}$, we have

Proposition 3.3. For $j \in\{1, \ldots, s\}$, one has:

(i) the sequence $\sqrt{n}\left(P_{j}^{(n)}-P_{j}\right)$ converges in distribution, as $n \rightarrow+\infty$, to ar.v. having a centered normal distibution in $\mathcal{L}\left(\mathcal{X}_{1}\right)$ with covariance operator $\Lambda_{j}=\Psi_{j} \Gamma \Psi_{j}^{*}$.

(ii) the sequence $\left(\sqrt{n}\left(\lambda_{i}^{(n)}-\lambda_{j}^{\prime}\right)\right)_{\nu_{j} \leq i \leq \nu_{j}+m_{j}-1}$ converges in distribution, as $n \rightarrow$ $+\infty$, to $\Delta\left(\xi_{j}\right)$, where $\xi_{j}$ is a r.v. having a centered normal distribution in $\mathcal{L}\left(\mathcal{X}_{1}\right)$ with covariance operator $\Lambda_{j}^{\prime}=\Psi_{j}^{\prime} \Gamma \Psi_{j}^{\prime *}$.

(iii) If $\rho_{j}^{\prime} \neq 0$, then $\left(\sqrt{n}\left(\rho_{i}^{(n)}-\rho_{j}^{\prime}\right)\right)_{\nu_{j} \leq i \leq \nu_{j}+m_{j}-1}$ converges in distribution, as $n \rightarrow$ $+\infty$, to $\Delta\left(\xi_{j}^{\prime}\right)$, where $\xi_{j}^{\prime}$ is a r.v. having a centered normal distribution in $\mathcal{L}\left(\mathcal{X}_{1}\right)$ with covariance operator $\Lambda_{j}^{\prime \prime}=\left(4 \lambda_{j}^{\prime}\right)^{-1} \Psi_{j}^{\prime} \Gamma \Psi_{j}^{\prime *}$.

(iv) If $m_{j}=1$, then $\sqrt{n}\left(\lambda_{\nu_{j}}^{(n)}-\lambda_{j}^{\prime}\right)$ converges in distribution, as $n \rightarrow+\infty$, to $\operatorname{tr}\left(\xi_{j}\right)$, and for $j$ satisfying $\rho_{j}^{\prime} \neq 0, \sqrt{n}\left(\rho_{i}^{(n)}-\rho_{j}^{\prime}\right)$ converges in distribution, as $n \rightarrow+\infty$, to $\operatorname{tr}\left(\xi_{j}^{\prime}\right)$.

(v) If $m_{j}=1$, then $\sqrt{n}\left(\alpha_{1 \cdot 3, n}^{\left(\nu_{j}\right)}-\alpha_{1 \cdot 3}^{\left(\nu_{j}\right)}\right)$ converges in distribution, as $n \rightarrow+\infty$, to a r.v. having a centered normal distribution in $\mathcal{X}_{1}$ with covariance operator $\Lambda_{j}^{\prime \prime}=\Theta_{j} \Gamma \Theta_{j}^{*}$. 
Proof. (i) and (ii). From Proposition 4 in Dossou-Gbete and Pousse (1991), we know that $\sqrt{n}\left(P_{j}^{(n)}-P_{j}\right)$ (resp. $\left.\left(\sqrt{n}\left(\lambda_{i}^{(n)}-\lambda_{j}^{\prime}\right)\right)_{\nu_{j} \leq i \leq \nu_{j}+m_{j}-1}\right)$ converges in distribution, as $n \rightarrow+\infty$, to $\Psi_{j}(U)$ (resp. $\Delta\left(\xi_{j}\right)$, where $\xi_{j}:=\Psi_{j}^{\prime}(U)$ ). Since $\Psi_{j}(U)$ (resp. $\xi_{j}$ ) is a linear function of $U$, then it has a centered normal distribution. Its covariance operator are easily shown to be $\Lambda_{j}$ (resp. $\Lambda_{j}^{\prime}$ ).

(iii) If $\rho_{j}^{\prime} \neq 0$, then from the equality

$$
\sqrt{n}\left(\rho_{i}^{(n)}-\rho_{j}^{\prime}\right)=\sqrt{n}\left(\lambda_{i}^{(n)}-\lambda_{j}^{\prime}\right)\left(\rho_{i}^{(n)}+\rho_{j}^{\prime}\right)^{-1}
$$

we can write $\left(\sqrt{n}\left(\rho_{i}^{(n)}-\rho_{j}^{\prime}\right)\right)_{\nu_{j} \leq i \leq \nu_{j}+m_{j}-1}=B_{j}^{(n)}\left(\eta_{j}^{(n)}\right)$ where $\eta_{j}^{(n)}:=\left(\sqrt{n}\left(\lambda_{i}^{(n)}-\right.\right.$ $\left.\left.\lambda_{j}^{\prime}\right)\right)_{\nu_{j} \leq i \leq \nu_{j}+m_{j}-1}$ and $B_{j}^{(n)}$ is the random operator

$$
\left(x_{i}\right)_{\nu_{j} \leq i \leq \nu_{j}+m_{j}-1} \in \mathbb{R}^{m_{j}} \mapsto\left(x_{i}\left(\rho_{i}^{(n)}+\rho_{j}^{\prime}\right)^{-1}\right)_{\nu_{j} \leq i \leq \nu_{j}+m_{j}-1} \in \mathbb{R}^{m_{j}} .
$$

For $i \in\left\{\nu_{j}, \ldots, \nu_{j}+m_{j}-1\right\}, \rho_{i}^{(n)}$ converges almost surely, as $n \rightarrow+\infty$, to $\rho_{j}^{\prime}$, then $B_{j}^{(n)}$ converges almost surely uniformly to the operator $B_{j}:=\left(2 \rho_{j}^{\prime}\right)^{-1} I_{\mathbb{R}^{m_{j}}}$, where $I_{\mathbb{R}^{m_{j}}}$ denotes the identity of $\mathbb{R}^{m_{j}}$. Moreover, we have:

$$
\left\|B_{j}^{(n)}\left(\eta_{j}^{(n)}\right)-B_{j}\left(\eta_{j}^{(n)}\right)\right\|_{\mathbb{R}^{m_{j}}} \leq\left\|B_{j}^{(n)}-B_{j}\right\|_{\infty}\left\|\eta_{j}^{(n)}\right\|_{\mathbb{R}^{m_{j}}}
$$

Since $\left\|\eta_{j}^{(n)}\right\|_{\mathbb{R}^{m_{j}}}$ (resp. $\left\|B_{j}^{(n)}-B_{j}\right\|_{\infty}$ ) converges in distribution (resp. in probability), as $n \rightarrow+\infty$, to $\left\|\Delta\left(\xi_{j}\right)\right\|_{\mathbb{R}^{m_{j}}}$ (resp. 0 ), the previous inequality implies the convergence in probability of $B_{j}^{(n)}\left(\eta_{j}^{(n)}\right)-B_{j}\left(\eta_{j}^{(n)}\right)$ to 0 as $n \rightarrow+\infty$. Hence $B_{j}^{(n)}\left(\eta_{j}^{(n)}\right)$ and $B_{j}\left(\eta_{j}^{(n)}\right)$ have the same limit distribution; using (ii) and the continuity of $B_{j}$, we then conclude that $\left(\sqrt{n}\left(\rho_{i}^{(n)}-\rho_{j}^{\prime}\right)\right)_{\nu_{j} \leq i \leq \nu_{j}+m_{j}-1}$ converges in distribution, as $n \rightarrow+\infty$, to $\Delta\left(\xi_{j}^{\prime}\right)$ where $\xi_{j}^{\prime}:=\left(2 \rho_{j}^{\prime}\right)^{-1} \xi_{j}$. Clearly, $\xi_{j}^{\prime}$ has a centered normal distribution with covariance operator $\left(4 \lambda_{j}^{\prime}\right)^{-1} \Psi_{j}^{\prime} \Gamma \Psi_{j}^{\prime *}$

(iv) and (v). If $m_{j}=1$, then $\xi_{j}$ and $\xi_{j}^{\prime}$ have ranks equal to one then $\Delta\left(\xi_{j}\right)=\operatorname{tr}\left(\xi_{j}\right)$ and $\Delta\left(\xi_{j}^{\prime}\right)=\operatorname{tr}\left(\xi_{j}^{\prime}\right)$. Moreover, we know from Proposition 4 in Dossou-Gbete and Pousse (1991) that $\sqrt{n}\left(\alpha_{1 \cdot 3, n}^{\left(\nu_{j}\right)}-\alpha_{1.3}^{\left(\nu_{j}\right)}\right)$ converges in distribution, as $n \rightarrow+\infty$, to $\Theta_{j}(U)$, that is a centered normal r.v. with covariance operator $\Lambda_{j}^{\prime \prime}=\Theta_{j} \Gamma \Theta_{j}^{*}$.

The covariance operator $\Gamma$ can be expressed using the canonical variates and coefficients related to the LRCA. For $(i, j) \in\left\{1, \ldots, p_{1}\right\}^{2}$, put

and

$$
\varepsilon_{i j \cdot 3}=\alpha_{1 \cdot 3}^{(i)} \otimes \alpha_{1 \cdot 3}^{(j)}+\alpha_{1 \cdot 3}^{(j)} \otimes \alpha_{1 \cdot 3}^{(i)}
$$

$$
F_{i j \cdot 3}=-\rho_{i}^{2} f_{1 \cdot 3}^{(i)} f_{1 \cdot 3}^{(j)}-\rho_{i} \rho_{j} f_{1 \cdot 3}^{(i)} f_{2 \cdot 3}^{(j)}+2 \rho_{j} f_{1 \cdot 3}^{(i)} f_{2 \cdot 3}^{(j)}
$$

(with $\rho_{i}=0$ if $i>r$ ); we then obtain

Corollary 3.1. One has: $\Gamma=\frac{1}{4} \sum_{1 \leq i, j, k, l \leq p_{1}} \mathbb{E}\left(F_{i j \cdot 3} F_{k l \cdot 3}\right) \varepsilon_{i j \cdot 3} \widetilde{\otimes} \varepsilon_{k l \cdot 3}$.

Proof. From (P2) and (P3) (see Section 2), it is easily seen that if $i \leq r$, $V_{12 \cdot 3} \alpha_{2 \cdot 3}^{(i)}=\rho_{i} \alpha_{1 \cdot 3}^{(i)}$ and that if $i>r, V_{12 \cdot 3} \alpha_{2 \cdot 3}^{(i)}=0$ (because $V_{21 \cdot 3} V_{12 \cdot 3} \alpha_{2 \cdot 3}^{(i)}=0$ ). Then, using equation (2.8), we obtain:

$$
X_{2 \cdot 3} \otimes X_{1 \cdot 3}=\sum_{i=1}^{p_{1}} \sum_{j=1}^{p_{2}} f_{1 \cdot 3}^{(i)} f_{2 \cdot 3}^{(j)} \alpha_{2 \cdot 3}^{(j)} \otimes \alpha_{1 \cdot 3}^{(i)}=\left(X_{1 \cdot 3} \otimes X_{2 \cdot 3}\right)^{*}
$$


and for $k \in\{1,2\}$ :

$$
X_{k \cdot 3}^{\otimes 2}=\sum_{i=1}^{p_{k}} \sum_{j=1}^{p_{k}} f_{k \cdot 3}^{(i)} f_{k \cdot 3}^{(j)} \alpha_{k \cdot 3}^{(i)} \otimes \alpha_{k \cdot 3}^{(j)}
$$

Thus, using equation (2.9), we obtain

$$
\begin{aligned}
& V_{12 \cdot 3}=\mathbb{E}\left(X_{2 \cdot 3} \otimes X_{1 \cdot 3}\right)=\sum_{i=1}^{p_{1}} \sum_{j=1}^{p_{2}} \delta_{i j} \rho_{i} \alpha_{2 \cdot 3}^{(j)} \otimes \alpha_{1 \cdot 3}^{(i)}=\sum_{i=1}^{r} \rho_{i} \alpha_{2 \cdot 3}^{(i)} \otimes \alpha_{1 \cdot 3}^{(i)}, \\
& V_{12 \cdot 3} V_{21 \cdot 3}=\sum_{i=1}^{r} \rho_{i}^{2} \alpha_{1 \cdot 3}^{(i)^{\otimes^{2}}}
\end{aligned}
$$

and using again equation (2.8)

$$
\begin{aligned}
V_{12 \cdot 3} V_{21 \cdot 3} X_{1 \cdot 3}^{\otimes^{2}} & =\sum_{i=1}^{r} \sum_{1 \leq j, k \leq p_{1}} \rho_{i}^{2} f_{1 \cdot 3}^{(j)} f_{1 \cdot 3}^{(k)} \alpha_{1 \cdot 3}^{(i)^{\otimes^{2}}} \alpha_{1 \cdot 3}^{(j)} \otimes \alpha_{1 \cdot 3}^{(k)} \\
& =\sum_{1 \leq i, k \leq r} \sum_{j=1}^{p_{1}} \rho_{i}^{2} f_{1 \cdot 3}^{(j)} f_{1 \cdot 3}^{(k)} \delta_{k i} \alpha_{1 \cdot 3}^{(j)} \otimes \alpha_{1 \cdot 3}^{(i)} \\
& =\sum_{i=1}^{r} \sum_{j=1}^{p_{1}} \rho_{i}^{2} f_{1 \cdot 3}^{(i)} f_{1 \cdot 3}^{(j)} \alpha_{1 \cdot 3}^{(j)} \otimes \alpha_{1 \cdot 3}^{(i)}
\end{aligned}
$$

moreover

$$
\begin{aligned}
V_{12 \cdot 3} X_{2 \cdot 3}^{\otimes^{2}} V_{21 \cdot 3} & =\left(V_{12 \cdot 3} X_{2 \cdot 3}\right)^{\otimes^{2}}=\left(\sum_{i=1}^{p_{1}} \sum_{j=1}^{p_{2}} \rho_{i} f_{2 \cdot 3}^{(j)}\left(\alpha_{2 \cdot 3}^{(i)} \otimes \alpha_{1 \cdot 3}^{(i)}\right) \alpha_{2 \cdot 3}^{(j)}\right)^{\otimes^{2}} \\
& =\left(\sum_{i=1}^{r} \rho_{i} f_{2 \cdot 3}^{(i)} \alpha_{1 \cdot 3}^{(i)}\right)^{\otimes^{2}}=\sum_{1 \leq i, j \leq r} \rho_{i} \rho_{j} f_{2 \cdot 3}^{(i)} f_{2 \cdot 3}^{(j)} \alpha_{1 \cdot 3}^{(i)} \otimes \alpha_{1 \cdot 3}^{(j)}
\end{aligned}
$$

and

$$
\begin{aligned}
V_{12 \cdot 3}\left(X_{1 \cdot 3} \otimes X_{2 \cdot 3}\right) & =\sum_{i=1}^{r} \sum_{j=1}^{p_{1}} \sum_{k=1}^{p_{2}} \rho_{i} f_{1 \cdot 3}^{(j)} f_{2 \cdot 3}^{(k)}\left(\alpha_{2 \cdot 3}^{(i)} \otimes \alpha_{1 \cdot 3}^{(i)}\right)\left(\alpha_{1 \cdot 3}^{(j)} \otimes \alpha_{2 \cdot 3}^{(k)}\right) \\
& =\sum_{1 \leq i, j \leq r} \rho_{j} f_{1 \cdot 3}^{(i)} f_{2 \cdot 3}^{(j)} \alpha_{1 \cdot 3}^{(i)} \otimes \alpha_{1 \cdot 3}^{(j)}
\end{aligned}
$$

Thus

$$
\Gamma=\mathbb{E}\left[\left(\pi\left(\sum_{1 \leq i, j \leq r} F_{i j} \alpha_{1 \cdot 3}^{(i)} \otimes \alpha_{1 \cdot 3}^{(j)}\right)\right)^{\tilde{\otimes}^{2}}\right]=\mathbb{E}\left[\left(\pi\left(\sum_{1 \leq i, j \leq p_{1}} F_{i j} \alpha_{1 \cdot 3}^{(i)} \otimes \alpha_{1 \cdot 3}^{(j)}\right)\right)^{\tilde{\otimes}^{2}}\right],
$$

the second equality being justified by the fact that if $i>r$ or $j>r$ then $F_{i j}=0$. Noticing that $\pi\left(\alpha_{1 \cdot 3}^{(i)} \otimes \alpha_{1 \cdot 3}^{(j)}\right)=\frac{1}{2} \varepsilon_{i j \cdot 3}$, one sees that the required result is obtained. 


\subsection{Asymptotic study of the elements associated with the null eigenvalue}

As it was noticed for the usual LCA (see Larrère (1994)), the previous results can not be exploited for statistical inference involving only the eigenelements associated with the null eigenvalue of $T_{1 \cdot 3}$. Indeed, easy calculations show that the eigenvalue limiting distributions obtained in Proposition 3.3 are Dirac distributions. Then it is necessary to use another approach for having the asymptotic distributions related to these associated eigenelements. Notice that we implicitly suppose $r<p_{1}$ (else there does not exist a null eigenvalue).

Let $P_{0}$ be the orthogonal projector onto the eigenspace associated with the null eigenvalue of $T_{1 \cdot 3}$, we have

$$
P_{0}=\sum_{i=r+1}^{p_{1}} \alpha_{1 \cdot 3}^{(i)^{\otimes^{2}}}
$$

and put $P_{0}^{(n)}:=\sum_{i=r+1}^{p_{1}} \alpha_{1 \cdot 3, n}^{(i)} \otimes^{2}$; from the Assertion (ii) of Proposition 3.1 we have the almost sure uniform convergence of $P_{0}^{(n)}$ to $P_{0}$, as $n \rightarrow+\infty$. Then $P_{0}^{(n)} T_{1 \cdot 3}^{(n)} P_{0}^{(n)}$ almost surely uniformly converges to $P_{0} T_{1 \cdot 3} P_{0}=0$, as $n \rightarrow+\infty$. Now, we can derive the limit distribution of $n P_{0}^{(n)} T_{1 \cdot 3}^{(n)} P_{0}^{(n)}$. Putting $P_{0}^{\prime}:=\sum_{i=r+1}^{p_{2}} \alpha_{2 \cdot 3}^{(i) \otimes^{2}}$ we have

Proposition 3.4. The r.v. $n P_{0}^{(n)} T_{13}^{(n)} P_{0}^{(n)}$ converges in distribution, as $n \rightarrow+\infty$, to $\Psi \Psi^{*}$, where $\Psi$ is a r.v. having a centered normal distribution in $\mathcal{L}\left(\mathcal{X}_{2}, \mathcal{X}_{1}\right)$, with covariance operator:

$$
\Gamma_{0}=\mathbb{E}\left[\left(P_{0}\left(X_{2 \cdot 3} \otimes X_{1 \cdot 3}\right) P_{0}^{\prime}\right)^{\tilde{\otimes}^{2}}\right]
$$

Proof. We have $n P_{0}^{(n)} T_{1 \cdot 3}^{(n)} P_{0}^{(n)}=\Psi_{n} \Psi_{n}^{*}$, with $\Psi_{n}=\sqrt{n} P_{0}^{(n)} V_{1 \cdot 3}^{(n)^{-1 / 2}} V_{12 \cdot 3}^{(n)}$. $V_{2 \cdot 3}^{(n)^{-1 / 2}}$. Using equation (3.8) and the orthonormality of the $\alpha_{1 \cdot 3}^{(i)}$ 's, we obtain $P_{0} V_{12 \cdot 3}=$ 0 . Thus

$$
\begin{aligned}
\Psi_{n}= & \sqrt{n}\left(P_{0}^{(n)}-P_{0}\right) V_{1 \cdot 3}^{(n)^{-1 / 2}} V_{12 \cdot 3}^{(n)} V_{2 \cdot 3}^{(n)^{-1 / 2}} \\
& +P_{0}\left[\sqrt{n}\left(V_{1 \cdot 3}^{(n)^{-1 / 2}}-I_{1}\right)\right] V_{12 \cdot 3}^{(n)} V_{2 \cdot 3}^{(n)^{-1 / 2}} \\
& +P_{0}\left[\sqrt{n}\left(V_{12 \cdot 3}^{(n)}-V_{12 \cdot 3}\right)\right] V_{2 \cdot 3}^{(n)^{-1 / 2}} \\
& +P_{0} V_{12 \cdot 3}\left[\sqrt{n}\left(V_{2 \cdot 3}^{(n)^{-1 / 2}}-I_{2}\right)\right]
\end{aligned}
$$

then using the relation in equation (3.6) with $T=V_{k \cdot 3}^{(n)}(k=1,2)$ and the Lemmas 3.1 and 3.2 , we have

$$
\Psi_{n}=\sqrt{n}\left(P_{0}^{(n)}-P_{0}\right) V_{1 \cdot 3}^{(n)^{-1 / 2}} V_{12 \cdot 3}^{(n)} V_{2 \cdot 3}^{(n)^{-1 / 2}}+\eta^{(n)}\left(H_{n}\right)
$$

where $\eta^{(n)}$ is the random operator

$$
\begin{aligned}
\eta^{(n)}(T)= & -P_{0} V_{1 \cdot 3}^{(n)^{-1 / 2}} a_{1 \cdot 3}^{(n)}(T)\left(V_{1 \cdot 3}^{(n)^{-1 / 2}}+I_{1}\right)^{-1} V_{12 \cdot 3}^{(n)} V_{2 \cdot 3}^{(n)^{-1 / 2}}+P_{0} a_{12 \cdot 3}^{(n)}(T) V_{2 \cdot 3}^{(n)^{-1 / 2}} \\
& -P_{0} V_{12 \cdot 3} V_{2 \cdot 3}^{(n)^{-1 / 2}} a_{2 \cdot 3}^{(n)}(T)\left(V_{2 \cdot 3}^{(n)^{-1 / 2}}+I_{1}\right)^{-1}
\end{aligned}
$$


which converges almost surely uniformly to the operator $\eta$ defined as

$$
T \in M \mapsto-\frac{1}{2} P_{0} a_{1 \cdot 3}(T) V_{12 \cdot 3}-\frac{1}{2} P_{0} V_{12 \cdot 3} a_{2 \cdot 3}(T)+P_{0} a_{12 \cdot 3}(T) \in \mathcal{L}\left(\mathcal{X}_{2}, \mathcal{X}_{1}\right)
$$

One knows that (see Dossou-Gbete and Pousse $(1991)) \sqrt{n}\left(P_{0}^{(n)}-P_{0}\right)=\phi^{(n)}\left(\sqrt{n}\left(T_{1 \cdot 3}^{(n)}-\right.\right.$ $\left.T_{1 \cdot 3}\right)$ ), where $\phi^{(n)}$ converges almost surely uniformly to

$$
\phi: T \in \mathcal{L}\left(\mathcal{X}_{1}\right) \mapsto P_{0} T S_{0}+S_{0} T P_{0} \in \mathcal{L}\left(\mathcal{X}_{1}\right)
$$

and $S_{0}:=-\sum_{i=1}^{r} \rho_{i}^{-2} \alpha_{1 \cdot 3}^{(i)^{\otimes^{2}}}$. As $\sqrt{n}\left(T_{1 \cdot 3}^{(n)}-T_{1 \cdot 3}\right)=\varphi_{n}\left(H_{n}\right)$ where $\varphi_{n}$ converges almost surely uniformly to $\varphi$ (see the proof of Proposition 3.2), $\Psi_{n}$ converges in distribution to $\eta^{\prime}(H)$ where

$$
\eta^{\prime}(T)=\phi(\varphi(T))+\eta(T)=P_{0} \varphi(T) S_{0}+S_{0} \varphi(T) P_{0}+\eta(T)
$$

since $\eta^{\prime}$ is linear, $\eta^{\prime}(H)$ has a centered normal distribution in $\mathcal{L}\left(\mathcal{X}_{1}\right)$. Its covariance operator is

$$
\Gamma_{0}=\eta^{\prime} \mathbb{E}\left[H^{\dot{\otimes}^{2}}\right] \eta^{\prime^{*}}=\eta^{\prime} \mathbb{E}\left[\left(Z^{\otimes^{2^{c}}}\right)^{\tilde{\otimes}^{2}}\right]{\eta^{\prime}}^{*}=\mathbb{E}\left[\left(\eta^{\prime}\left(Z^{\otimes^{2^{c}}}\right)\right)^{\tilde{\otimes}^{2}}\right]
$$

(recall that $Z^{\otimes^{2^{c}}}=Z^{\otimes^{2}}-\mathbb{E}\left(Z^{\otimes^{2}}\right)$ ). Using equation (3.8) and the orthonormality of the $\alpha_{k \cdot 3}^{(i)}$ 's $(k=1,2)$ implies $V_{21 \cdot 3} S_{0} V_{12 \cdot 3}=P_{0}^{\prime}-I_{2}$. From equations (3.7) and (3.9), and recalling that $P_{0} V_{12 \cdot 3}=0$, we obtain $\eta\left(Z^{\otimes^{2^{c}}}\right)=P_{0}\left(X_{2 \cdot 3} \otimes X_{1 \cdot 3}\right) P_{0}^{\prime}$; this completes the proof.

\section{Some applications}

\subsection{Testing for the lack of linear relative association}

LRCA may be seen as a tool which permits to see if there is or not a linear relative association between $X_{1}$ and $X_{2}$ relative to $X_{3}$. We say that there is a lack of linear relative association, if

$$
V_{12}=V_{13} V_{3}^{-1} V_{32}
$$

that is $V_{12.3}=0$. One knows that (see, e.g., Timm and Carlson (1976)) when $\left(X_{1}, X_{2}, X_{3}\right)$ has a normal distribution this property is equivalent to the conditional independence of $X_{1}$ and $X_{2}$, given $X_{3}$.

Following an approach which has been used for classical LCA (Cramer and Nicewander (1979), Lin (1987), Cléroux and Lazraq (1988), Dauxois and Nkiet (1997b), Nkiet (2000)), a class of linear relative association measures have been introduced by Dauxois and Nkiet (2002). These measures have the form $m_{/ X_{3}}\left(X_{1}, X_{2}\right):=\Phi(\lambda)$, where $\Phi$ is a continuous symmetric function from $\mathbb{R}^{p_{1}}$ to $\mathbb{R}_{+}$satisfying $\Phi(x)=0 \Leftrightarrow x=0$. These measures can be used for testing for lack of linear relative association since equation (4.1) is equivalent to $m_{/ X_{3}}\left(X_{1}, X_{2}\right)=0$ (see Dauxois and Nkiet (2002)). Then we consider the test of $H_{0}: m_{/ X_{3}}\left(X_{1}, X_{2}\right)=0$ against $H_{1}: m_{/ X_{3}}\left(X_{1}, X_{2}\right)>0$.

We take as test statistic, the r.v. $\Phi\left(\lambda^{(n)}\right)$ because, by (i) of Proposition 3.1 and the continuity of $\Phi$, it is a strongly consistent estimator of $m_{/ X_{3}}\left(X_{1}, X_{2}\right)$. In order to derive the asymptotic distribution of $\Phi\left(\lambda^{(n)}\right)$ under $H_{0}$, we suppose that the following assumptions hold: 
(A5) $\Phi$ is twice differentiable on an open set $\mathcal{O}$ containing $\lambda$ and $\lambda^{(n)}(\omega)(\omega \in \Omega)$, and it exists $M>0$ such that for any $x \in \mathcal{O}$, one has $\left\|D^{2} \Phi(x)\right\| \leq M$;

(A6) putting $K_{\Phi}:=\frac{\partial \Phi}{\partial x_{1}}(0)$, one has $K_{\Phi} \neq 0$.

Since under $H_{0}$ one has $s=1, \lambda_{1}^{\prime}=0, P_{0}^{\prime}=I_{2} P_{1}=P_{0}=P_{0}^{(n)}=I_{1}$, a direct application of Theorem 1 of Dauxois and Nkiet (2000) and Proposition 3.4 gives

Proposition 4.1. Under $H_{0}, n K_{\Phi}^{-1} \Phi\left(\lambda^{(n)}\right)$ converges in distribution, as $n \rightarrow$ $+\infty$, to $R:=\operatorname{tr}\left(\Psi \Psi^{*}\right)$, where $\Psi$ has a centered normal distribution in $\mathcal{L}\left(\mathcal{X}_{\mathbf{2}}, \mathcal{X}_{\mathbf{1}}\right)$ with covariance operator equal to $\Gamma_{0}=\mathbb{E}\left[\left(X_{2 \cdot 3} \otimes X_{1 \cdot 3}\right)^{\tilde{\otimes}^{2}}\right]$.

Then, for a given asymptotic level $\alpha \in] 0,1$, the null hypothesis is rejected when $n K_{\Phi}^{-1} \Phi\left(\lambda^{(n)}\right)>\mathbb{F}_{Q}^{-1}(\alpha)$, where $\mathbb{F}_{Q}$ is the distribution function of $Q$. Note that $Q$ is a regular quadratic form of normal vector; then since $\mathbb{F}_{Q}$ is continuous (see Mathai and Provost (1992)), it is a bijective function. When $\left(X_{1}, X_{2}, X_{3}\right)$ has an elliptic distribution, $Q$ is of a more simpler form:

COROllary 4.1. If $\left(X_{1}, X_{2}, X_{3}\right)$ has an elliptic distribution with kurtosis $\kappa$ then under $H_{0}$ the distribution of $R$ is $(1+\kappa) \chi_{p_{1} p_{2}}^{2}$.

Proof. Since $\left(\alpha_{1 \cdot 3}^{(i)}\right)_{1 \leq i \leq p_{1}}$ (resp. $\left.\left(\alpha_{2 \cdot 3}^{(j)}\right)_{1 \leq j \leq p_{j}}\right)$ is an orthonormal basis of $\mathcal{X}_{1}$ (resp. $\left.\mathcal{X}_{2}\right)$, we can write

$$
\Psi=\sum_{i=1}^{p_{1}} \sum_{j=1}^{p_{2}}\left\langle\Psi, \alpha_{2 \cdot 3}^{(j)} \otimes \alpha_{1 \cdot 3}^{(i)}\right\rangle_{2} \alpha_{2 \cdot 3}^{(j)} \otimes \alpha_{1 \cdot 3}^{(i)}
$$

thus, putting $W_{i j}:=\left\langle\Psi, \alpha_{2 \cdot 3}^{(j)} \otimes \alpha_{1.3}^{(i)}\right\rangle_{2}$ :

$$
\begin{aligned}
\operatorname{tr}\left(\Psi \Psi^{*}\right) & =\sum_{\substack{1 \leq i, k \leq p_{1} \\
1 \leq j, l \leq p_{2}}} W_{i j} W_{k l} \operatorname{tr}\left(\left(\alpha_{2 \cdot 3}^{(j)} \otimes \alpha_{1 \cdot 3}^{(i)}\right)\left(\alpha_{1 \cdot 3}^{(k)} \otimes \alpha_{2 \cdot 3}^{(l)}\right)\right) \\
& =\sum_{i=1}^{p_{1}} \sum_{j=1}^{p_{2}} W_{i j}^{2}
\end{aligned}
$$

the later equality being obtained from the properties $\left(\alpha_{2 \cdot 3}^{(j)} \otimes \alpha_{1 \cdot 3}^{(i)}\right)\left(\alpha_{1 \cdot 3}^{(k)} \otimes \alpha_{2 \cdot 3}^{(l)}\right)=\delta_{l j} \alpha_{1 \cdot 3}^{(k)} \otimes$ $\alpha_{1 \cdot 3}^{(i)}$ and $\operatorname{tr}\left(\alpha_{1 \cdot 3}^{(k)} \otimes \alpha_{1 \cdot 3}^{(i)}\right)=\left\langle\alpha_{1 \cdot 3}^{(k)}, \alpha_{1 \cdot 3}^{(i)}\right\rangle_{\mathcal{X}_{1}}=\delta_{k i}$. Each $W_{i j}$ has a centered normal distribution because it is a linear function of $\Psi$. Moreover, for any $(i, k, j, l) \in\left\{1, \ldots, p_{1}\right\}^{2} \times$ $\left\{1, \ldots, p_{2}\right\}^{2}$ we have

$$
\begin{aligned}
\mathbb{E}\left(W_{i j} W_{k l}\right) & =\mathbb{E}\left(\left\langle\Psi, \alpha_{2 \cdot 3}^{(j)} \otimes \alpha_{1 \cdot 3}^{(i)}\right\rangle_{2}\left\langle\Psi, \alpha_{2 \cdot 3}^{(l)} \otimes \alpha_{1 \cdot 3}^{(k)}\right\rangle_{2}\right) \\
& =\mathbb{E}\left(\left\langle(\Psi \widetilde{\otimes} \Psi)\left(\alpha_{2 \cdot 3}^{(j)} \otimes \alpha_{1 \cdot 3}^{(i)}\right), \alpha_{2 \cdot 3}^{(l)} \otimes \alpha_{1 \cdot 3}^{(k)}\right\rangle_{2}\right) \\
& =\left\langle\Gamma_{0}\left(\alpha_{2 \cdot 3}^{(j)} \otimes \alpha_{1 \cdot 3}^{(i)}\right), \alpha_{2 \cdot 3}^{(l)} \otimes \alpha_{1 \cdot 3}^{(k)}\right\rangle_{2} .
\end{aligned}
$$

Furthermore

$$
\Gamma_{0}\left(\alpha_{2 \cdot 3}^{(j)} \otimes \alpha_{1 \cdot 3}^{(i)}\right)=\mathbb{E}\left(\left\langle X_{2 \cdot 3} \otimes X_{1 \cdot 3}, \alpha_{2 \cdot 3}^{(j)} \otimes \alpha_{1 \cdot 3}^{(i)}\right\rangle_{2}\left(X_{2 \cdot 3} \otimes X_{1 \cdot 3}\right)\right),
$$


hence

$$
\mathbb{E}\left(W_{i j} W_{k l}\right)=\mathbb{E}\left(\left\langle X_{2 \cdot 3} \otimes X_{1 \cdot 3}, \alpha_{2 \cdot 3}^{(j)} \otimes \alpha_{1 \cdot 3}^{(i)}\right\rangle_{2}\left\langle X_{2 \cdot 3} \otimes X_{1 \cdot 3}, \alpha_{2 \cdot 3}^{(l)} \otimes \alpha_{1 \cdot 3}^{(k)}\right\rangle_{2}\right)
$$

From the equalities $\langle x \otimes y, z \otimes t\rangle_{2}=\operatorname{tr}((x \otimes y)(t \otimes z))=\operatorname{tr}(\langle x, z\rangle(t \otimes y))$ and $\operatorname{tr}(t \otimes y)=\langle t, y\rangle$, it follows

$$
\mathbb{E}\left(W_{i j} W_{k l}\right)=\mathbb{E}\left(f_{1 \cdot 3}^{(i)} f_{1 \cdot 3}^{(k)} f_{2 \cdot 3}^{(j)} f_{2 \cdot 3}^{(l)}\right)
$$

where $f_{k \cdot 3}^{(i)}$ is defined in equation (2.7) (recall that $V_{1 \cdot 3}=I_{1}$ and $V_{2 \cdot 3}=I_{2}$ ). Since $\left(f_{1 \cdot 3}^{(i)}, f_{1 \cdot 3}^{(k)}, f_{2 \cdot 3}^{(j)}, f_{2 \cdot 3}^{(l)}\right)$ is a linear function of $\left(X_{1}, X_{2}, X_{3}\right)$, it also has an elliptic distribution with kurtosis $\kappa$. Hence

$$
\begin{aligned}
\mathbb{E}\left(W_{i j} W_{k l}\right)=(1+\kappa)\left[\mathbb{E}\left(f_{1 \cdot 3}^{(i)} f_{1 \cdot 3}^{(k)}\right) \mathbb{E}\left(f_{2 \cdot 3}^{(j)} f_{2 \cdot 3}^{(l)}\right)\right. & +\mathbb{E}\left(f_{1 \cdot 3}^{(i)} f_{2 \cdot 3}^{(j)}\right) \mathbb{E}\left(f_{1 \cdot 3}^{(k)} f_{2 \cdot 3}^{(l)}\right) \\
& \left.+\mathbb{E}\left(f_{1 \cdot 3}^{(i)} f_{2 \cdot 3}^{(l)}\right) \mathbb{E}\left(f_{1 \cdot 3}^{(k)} f_{2 \cdot 3}^{(j)}\right)\right]
\end{aligned}
$$

Under $H_{0}$, all the canonical coefficients are null since $V_{12.3}=0$; then using equations (2.9) and $(2.10)$ we obtain $\mathbb{E}\left(W_{i j} W_{k l}\right)=(1+\kappa)\left(\delta_{i k} \delta_{j l}\right)$. This shows that $\mathbb{E}\left(W_{i j}^{2}\right)=(1+\kappa)$ and if $(i, j) \neq(k, l), \mathbb{E}\left(W_{i j} W_{k l}\right)=0$; then the r.v. $(1+\kappa)^{-1} W_{i j}$ are independent and have the standard normal distribution. Using equation (4.2) we conclude that $R=(1+\kappa) \chi_{p_{1} p_{2}}^{2}$.

Remark 4.1. This approach for testing lack of linear relative association yields a general framework containing tests which are based on what is known in the literature as partial canonical correlation coefficients. For example, the test proposed by Timm and Carlson (1976) under the assumption that $\left(X_{1}, X_{2}, X_{3}\right)$ has a normal distribution appears in our context as a particular case by taking $\Phi(x)=-\sum_{i=1}^{p_{1}} \ln \left(1-x_{i}\right)$. Notice that when $p_{1}=1$ (resp. $p_{1}=p_{2}=1$ ) our test is based on a function of the classical partial multiple correlation coefficient (resp. partial correlation coefficient).

\subsection{Testing for dimensionality in LRCA}

As in Fujikoshi and Veitch (1979) for usual LCA, we can introduce tests for determining the dimensionality of LRCA, that is the integer $d \in\left\{1, \ldots, p_{1}\right\}$ equal to $p_{1}$ if all the relative canonical coefficients are non null or such that

$$
\lambda_{1} \geq \lambda_{2} \geq \cdots \geq \lambda_{d}>\lambda_{d+1}=\cdots=\lambda_{p_{1}}=0
$$

elsewhere. For doing that, we introduce for any $k \in\left\{1, \ldots, p_{1}-1\right\}$ a test for $H_{0}^{(k)}$ against $H_{1}^{(k)}$, with

and

$$
H_{0}^{(k)}: \lambda_{k}>\lambda_{k+1}=\cdots=\lambda_{p_{1}}=0
$$

$$
H_{1}^{(k)}: \lambda_{k}=0
$$

Notice $\lambda_{k+1}, \ldots, \lambda_{p_{1}}$ are the eigenvalues of $R_{1 \cdot 3}^{(k)}=Q_{k} T_{1 \cdot 3} Q_{k}$, where $Q_{k}:=$ $\sum_{i=k+1}^{p_{1}} \alpha_{1.3}^{(i)^{\otimes^{2}}}$. Then we take as test statistic the r.v. $\Phi\left(\mu_{k}^{(n)}\right)$, where

$$
\mu_{k}^{(n)}:=\left(\lambda_{k+1}^{(n)}, \ldots, \lambda_{p_{1}}^{(n)}\right)=\Delta\left(Q_{k}^{(n)} T_{1 \cdot 3}^{(n)} Q_{k}^{(n)}\right)
$$


with $Q_{k}^{(n)}:=\sum_{i=k+1}^{p_{1}} \alpha_{1 \cdot 3, n}^{(i)^{\otimes^{2}}}$, and $\Phi$ is a symmetric function from $\mathbb{R}^{p_{1}-k}$ to $\mathbb{R}$ satisfying (A5), (A6) and $\Phi(x)=0 \Leftrightarrow x=0$. Let us consider $Q_{k}^{\prime}:=\sum_{i=k+1}^{p_{1}} \alpha_{2 \cdot 3}^{(i)^{\otimes^{2}}}$. Under $H_{0}^{(k)}$, we obviously have $Q_{k}^{\prime}=P_{0}^{\prime}, Q_{k}=P_{0}$ and $Q_{k}^{(n)}=P_{0}^{(n)}$, hence by applying again Proposition 3.4 and Theorem 1 of Dauxois and Nkiet (2000) we obtain:

Proposition 4.2. Under $H_{0}^{(k)}, n K_{\Phi}^{-1} \Phi\left(\mu_{k}^{(n)}\right)$ converges in distribution, as $n \rightarrow$ $+\infty$, to $R_{k}:=\operatorname{tr}\left(\Psi_{k} \Psi_{k}^{*}\right)$, where $\Psi_{k}$ has a centered normal distribution in $\mathcal{L}\left(\mathcal{X}_{2}, \mathcal{X}_{1}\right)$ with covariance operator $\Gamma_{k}=\mathbb{E}\left[\left(Q_{k}\left(X_{2 \cdot 3} \otimes X_{1 \cdot 3}\right) Q_{k}^{\prime}\right)^{\tilde{\otimes}^{2}}\right]$.

Then, for a given asymptotic level $\alpha \in] 0,1[$, the null hypothesis is rejected when $n K_{\Phi}^{-1} \Phi\left(\lambda^{(n)}\right)>\mathbb{F}_{Q}^{-1}(\alpha)$, where $\mathbb{F}_{Q}$ is the distribution function of $Q$. For determining the dimensionality, we use the previous test for $k=p_{1}-1, k=p_{1}-2$ and so on until $H_{0}^{(k)}$ is not rejected or $k=1$ (in this case the dimensionality is 1 ).

When $\left(X_{1}, X_{2}, X_{3}\right)$ has an elliptic distribution we have:

COROLlary 4.2. Under $H_{0}^{(k)}$, if $\left(X_{1}, X_{2}, X_{3}\right)$ has an elliptic distribution with kurtosis $\kappa$, then the distribution of $R_{k}$ is $(1+\kappa) \chi_{\left(p_{1}-k\right)\left(p_{2}-k\right)}^{2}$.

Proof. By similar arguments as in the proof of Corollary 4.1, we have

$$
\operatorname{tr}\left(\Psi_{k} \Psi_{k}^{*}\right)=\sum_{i=1}^{p_{1}} \sum_{j=1}^{p_{2}} W_{i j}^{(k)^{2}}
$$

where $W_{i j}^{(k)}:=\left\langle\Psi_{k}, \alpha_{2 \cdot 3}^{(j)} \otimes \alpha_{1 \cdot 3}^{(i)}\right\rangle_{2}$. Each $W_{i j}^{(k)}$ has a centered normal distribution because it is a linear function of $\Psi_{k}$. Moreover, for any $(i, h, j, l) \in\left\{1, \ldots, p_{1}\right\}^{2} \times\left\{1, \ldots, p_{2}\right\}^{2}$ we have

$$
\begin{aligned}
\mathbb{E}\left(W_{i j}^{(k)} W_{h l}^{(k)}\right)= & \left\langle\Gamma_{k}\left(\alpha_{2 \cdot 3}^{(j)} \otimes \alpha_{1 \cdot 3}^{(i)}\right), \alpha_{2 \cdot 3}^{(l)} \otimes \alpha_{1 \cdot 3}^{(h)}\right\rangle_{2} \\
=\mathbb{E}( & \left\langle Q_{k}\left(X_{2 \cdot 3} \otimes X_{1 \cdot 3}\right) Q_{k}^{\prime}, \alpha_{2 \cdot 3}^{(j)} \otimes \alpha_{1 \cdot 3}^{(i)}\right\rangle_{2} \\
& \left.\quad \times\left\langle Q_{k}\left(X_{2 \cdot 3} \otimes X_{1 \cdot 3}\right) Q_{k}^{\prime}, \alpha_{2 \cdot 3}^{(l)} \otimes \alpha_{1 \cdot 3}^{(h)}\right\rangle_{2}\right) .
\end{aligned}
$$

Under $H_{0}^{(k)}$, the rank of $T_{1 \cdot 3}$ equals $k$, and $Q_{k}^{\prime}=P_{0}^{\prime}, Q_{k}=P_{0}$; then, since $P_{0} \alpha_{1 \cdot 3}^{(i)}=0$ (resp. $P_{0}^{\prime} \alpha_{2 \cdot 3}^{(j)}=0$ ) when $i \leq k$ (resp. $j \leq k$ ), we deduce from

$$
\begin{aligned}
& \left\langle Q_{k}\left(X_{2 \cdot 3} \otimes X_{1 \cdot 3}\right) Q_{k}^{\prime}, \alpha_{2 \cdot 3}^{(j)} \otimes \alpha_{1 \cdot 3}^{(i)}\right\rangle_{2} \\
& \quad=\operatorname{tr}\left(\left(X_{2 \cdot 3} \otimes X_{1 \cdot 3}\right) P_{0}^{\prime}\left(\alpha_{1 \cdot 3}^{(i)} \otimes \alpha_{2 \cdot 3}^{(j)}\right) P_{0}\right) \\
& \quad=\operatorname{tr}\left(\left(X_{2 \cdot 3} \otimes X_{1 \cdot 3}\right)\left(\left(P_{0} \alpha_{1 \cdot 3}^{(i)}\right) \otimes\left(P_{0}^{\prime} \alpha_{2 \cdot 3}^{(j)}\right)\right)\right)
\end{aligned}
$$

and equation (4.4) that if $i \leq k$ or $j \leq k$ then $\mathbb{E}\left(W_{i j}^{(k)^{2}}\right)=0$, that is $W_{i j}^{(k)}=0$. Further, if $i>k$ and $j>k$ then $P_{0} \alpha_{1 \cdot 3}^{(i)}=\alpha_{1 \cdot 3}^{(i)}$ and $P_{0}^{\prime} \alpha_{2 \cdot 3}^{(j)}=\alpha_{2 \cdot 3}^{(j)}$; hence, equation (4.5) implies

$$
\mathbb{E}\left(W_{i j} W_{h l}\right)=\mathbb{E}\left(f_{1 \cdot 3}^{(i)} f_{1 \cdot 3}^{(h)} f_{2 \cdot 3}^{(j)} f_{2 \cdot 3}^{(l)}\right)
$$


where $f_{k \cdot 3}^{(i)}$ is defined in equation (2.7) (recall that $V_{1 \cdot 3}=I_{1}$ and $V_{2 \cdot 3}=I_{2}$ ). Using similar arguments than in the proof of Corollary 4.1 , we obtain

$$
\begin{aligned}
\mathbb{E}\left(W_{i j} W_{h l}\right)=(1+\kappa)\left[\mathbb{E}\left(f_{1 \cdot 3}^{(i)} f_{1 \cdot 3}^{(h)}\right) \mathbb{E}\left(f_{2 \cdot 3}^{(j)} f_{2 \cdot 3}^{(l)}\right)\right. & +\mathbb{E}\left(f_{1 \cdot 3}^{(i)} f_{2 \cdot 3}^{(j)}\right) \mathbb{E}\left(f_{1 \cdot 3}^{(h)} f_{2 \cdot 3}^{(l)}\right) \\
& \left.+\mathbb{E}\left(f_{1 \cdot 3}^{(i)} f_{2 \cdot 3}^{(l)}\right) \mathbb{E}\left(f_{1 \cdot 3}^{(h)} f_{2 \cdot 3}^{(j)}\right)\right]
\end{aligned}
$$

Since $k=r$, equation (2.9) implies that for $j>k$, we have $\mathbb{E}\left(f_{1 \cdot 3}^{(i)} f_{2 \cdot 3}^{(j)}\right)=0$. Then using equation (2.10) we obtain $\mathbb{E}\left(W_{i j} W_{h l}\right)=(1+\kappa)\left(\delta_{i h} \delta_{j l}\right)$, that is $\mathbb{E}\left(W_{i j}^{2}\right)=(1+\kappa)$ and $\mathbb{E}\left(W_{i j} W_{k l}\right)=0$ if $(i, j) \neq(h, l)$. We conclude that if $i>k$ and $j>k$ then the r.v. $(1+\kappa)^{-1} W_{i j}$ are independent and have the standard normal distribution, and if $i \leq k$ or $j \leq k$ then they are null. The equation (4.3) can be rewritten as

$$
\operatorname{tr}\left(\Psi_{k} \Psi_{k}^{*}\right)=\sum_{i=k+1}^{p_{1}} \sum_{j=k+1}^{p_{2}} W_{i j}^{(k)^{2}}
$$

and this shows that $R_{k}=(1+\kappa) \chi_{\left(p_{1}-k\right)\left(p_{2}-k\right)}^{2}$.

\subsection{Testing for invariance of $L R C A$}

Invariance for LRCA when the related variables are transformed by linear maps have been defined in Section 2 and conditions for having this invariance property have been obtained. Nevetheless, since these conditions involve covariance operators which are unknown in practice, it is of interest to contruct a test which permits to see whether or not LRCA is invariant for a given pair of linear maps. Notice that such an approach has already been used in the literature. Indeed, in Fujikoshi (1982) and in Fujikoshi and Khatri (1990) likelihood ratio tests for additional information in LCA and for redundancy in covariate discriminant analysis under normal assumption were introduced. These tests just are particular tests for invariance when the related variables are transformed by projectors. More recently, a generalizing approach has been adopted by Nkiet (2003) who introduced a test for the invariance of LCA when the related variables are transformed by linear maps which may not be projectors, without other assumption on the distribution of these variables besides the existence of four order moments.

Here we extend for LRCA an approach used in Nkiet (2003) for LCA. Note that the results of this later work can not be applied for LRCA because we do not have an i.i.d. sample of $X_{1.3}$ and $X_{2.3}$; these r.v. are unobservable since their definitions involve covariance operators which are unknown in practice. Let $A_{1}$ and $A_{2}$ be linear maps defined on $\mathcal{X}_{1}$ and $\mathcal{X}_{2}$ respectively. Our purpose is to introduce a test for the invariance of the LRCA of $X_{1}$ and $X_{2}$ relative to $X_{3}$. Consider

$$
\begin{aligned}
& C_{1}\left(A_{1}\right)=\left\|V_{12 \cdot 3}-V_{1 \cdot 3} A_{1}^{*}\left(A_{1} V_{1 \cdot 3} A_{1}^{*}\right)^{\dagger} A_{1} V_{12 \cdot 3}\right\|^{2}, \\
& C_{2}\left(A_{2}\right)=\left\|V_{21 \cdot 3}-V_{2 \cdot 3} A_{2}^{*}\left(A_{2} V_{2 \cdot 3} A_{2}^{*}\right)^{\dagger} A_{2 \cdot 3} V_{21 \cdot 3}\right\|^{2}, \\
& C\left(A_{1}, A_{2}\right)=C_{1}\left(A_{1}\right)+C_{2}\left(A_{2}\right),
\end{aligned}
$$

where $\|\cdot\|$ denotes the norm associated with the Hilbert-Schmidt inner product. From Proposition 2.2 it is seen that the aforementioned test is the test of the hypothesis

$$
H_{0}: C\left(A_{1}, A_{2}\right)=0 \quad \text { against } \quad H_{1}: C\left(A_{1}, A_{2}\right)>0 .
$$


Defining for $(k, j) \in\{1,2\}^{2}$ with $k \neq j$

and

$$
\begin{aligned}
& S_{k \cdot 3}:=V_{k \cdot 3} A_{k}^{*}\left(A_{k} V_{k \cdot 3} A_{k}^{*}\right)^{\dagger} A_{k} V_{k j \cdot 3} \\
& S_{k \cdot 3}^{(n)}:=V_{k \cdot 3}^{(n)} A_{k}^{*}\left(A_{k} V_{k \cdot 3}^{(n)} A_{k}^{*}\right)^{\dagger} A_{k} V_{k j \cdot 3}^{(n)}
\end{aligned}
$$

$$
C_{k}^{(n)}\left(A_{k}\right)=\left\|V_{k j \cdot 3}^{(n)}-S_{k \cdot 3}^{(n)}\right\|^{2},
$$

we take as test statistic the estimator $C^{(n)}\left(A_{1}, A_{2}\right):=C_{1}^{(n)}\left(A_{1}\right)+C_{2}^{(n)}\left(A_{2}\right)$ of $C\left(A_{1}, A_{2}\right)$. For defining the corresponding (asymptotic) critical region, we must derive the limit distribution of $C^{(n)}\left(A_{1}, A_{2}\right)$ under $H_{0}$. Letting $\gamma_{1 \cdot 3}$ and $\gamma_{2 \cdot 3}$ be the operators from $M$ to $\mathcal{L}\left(\mathcal{X}_{2}, \mathcal{X}_{1}\right)$ and $\mathcal{L}\left(\mathcal{X}_{1}, \mathcal{X}_{2}\right)$ respectively, defined by

$$
\begin{aligned}
\gamma_{k \cdot 3}(T)= & a_{k \cdot 3}(T) A_{k}^{*}\left(A_{k} V_{k \cdot 3} A_{k}^{*}\right)^{\dagger} A_{k} V_{k j \cdot 3} \\
& -V_{k \cdot 3} A_{k}^{*}\left(A_{k} V_{k \cdot 3} A_{k}^{*}\right)^{\dagger} A_{k} a_{k \cdot 3}(T) A_{k}^{*}\left(A_{k} V_{k \cdot 3} A_{k}^{*}\right)^{\dagger} A_{k} V_{k j \cdot 3} \\
& +V_{k \cdot 3} A_{k}^{*}\left(A_{k} V_{k \cdot 3} A_{k}^{*}\right)^{\dagger} a_{k j \cdot 3}(T)-a_{k j \cdot 3}(T),
\end{aligned}
$$

where $(k, j) \in\{1,2\}^{2}$ with $k \neq j$, we have

Proposition 4.3. Under $H_{0}$, the r.v. $n C^{(n)}\left(A_{1}, A_{2}\right)$ converges in distribution, as $n \rightarrow+\infty$, to $Q=\left\|\gamma_{1 \cdot 3}(H)\right\|^{2}+\left\|\gamma_{2 \cdot 3}(H)\right\|^{2}$.

Proof. First, for $(k, j) \in\{1,2\}^{2}$ with $k \neq j$, we have:

$$
\begin{aligned}
\sqrt{n}\left(S_{k \cdot 3}^{(n)}-S_{k \cdot 3}\right)= & \sqrt{n}\left(V_{k \cdot 3}^{(n)}-V_{k \cdot 3}\right) A_{k}^{*}\left(A_{k} V_{k \cdot 3}^{(n)} A_{k}^{*}\right)^{\dagger} A_{k} V_{k j \cdot 3}^{(n)} \\
& +\sqrt{n} V_{k \cdot 3} A_{k}^{*}\left[\left(A_{k} V_{k \cdot 3}^{(n)} A_{k}^{*}\right)^{\dagger}-\left(A_{k} V_{k \cdot 3} A_{k}^{*}\right)^{\dagger}\right] A_{k} V_{k j \cdot 3}^{(n)} \\
& +\sqrt{n} V_{k \cdot 3} A_{k}^{*}\left(A_{k} V_{k \cdot 3} A_{k}^{*}\right)^{\dagger} A_{k}\left(V_{k j \cdot 3}^{(n)}-V_{k j \cdot 3}\right) .
\end{aligned}
$$

It is known that for any operators $T$ and $S$ one has

$$
T^{\dagger}-S^{\dagger}=-T^{\dagger}(T-S) S^{\dagger}+T^{\dagger^{2}}(T-S) \Pi_{\mathrm{ker}(S)}-\Pi_{\mathrm{ker}(T)}(T-S) S^{\dagger^{2}}
$$

(see Theorem 3.10 in Nashed (1976)); then applying this property with $T=A_{k} V_{k \cdot 3}^{(n)} A_{k}^{*}$ and $S=A_{k} V_{k \cdot 3} A_{k}^{*}$ and using equation (4.7), we obtain $\sqrt{n}\left(S_{k \cdot 3}^{(n)}-S_{k \cdot 3}\right)=\beta_{k \cdot 3}^{(n)}\left(H_{n}\right)$, where $\beta_{k \cdot 3}^{(n)}$ is the random variable valued into $\mathcal{L}\left(M, \mathcal{L}\left(\mathcal{X}_{3-k}, \mathcal{X}_{k}\right)\right)$ defined by

$$
\begin{aligned}
\beta_{k \cdot 3}^{(n)}(T)= & a_{k \cdot 3}^{(n)}(T) A_{k}^{*}\left(A_{k} V_{k \cdot 3}^{(n)} A_{k}^{*}\right)^{\dagger} A_{k} V_{k j \cdot 3}^{(n)} \\
& -V_{k \cdot 3} A_{k}^{*}\left(A_{k} V_{k \cdot 3}^{(n)} A_{k}^{*}\right)^{\dagger} A_{k} a_{k \cdot 3}^{(n)}(T) A_{k}^{*}\left(A_{k} V_{k \cdot 3} A_{k}^{*}\right)^{\dagger} A_{k} V_{k j \cdot 3}^{(n)} \\
& +V_{k \cdot 3} A_{k}^{*}\left(A_{k} V_{k \cdot 3}^{(n)} A_{k}^{*}\right)^{\dagger} A_{k} a_{k \cdot 3}^{(n)}(T) A_{k}^{*} \Pi_{\mathrm{ker}\left(A_{k} V_{k \cdot 3} A_{k}^{*}\right)} A_{k} V_{k j \cdot 3}^{(n)} \\
& -V_{k \cdot 3} A_{k}^{*} \Pi_{\operatorname{ker}\left(A_{k} V_{k \cdot 3}^{(n)} A_{k}^{*}\right)} A_{k} a_{k \cdot 3}^{(n)}(T) A_{k}^{*}\left(A_{k} V_{k \cdot 3} A_{k}^{*}\right)^{\dagger^{2}} A_{k} V_{k j \cdot 3}^{(n)} \\
& +V_{k \cdot 3} A_{k}^{*}\left(A_{k} V_{k \cdot 3} A_{k}^{*}\right)^{\dagger} A_{k} a_{k j \cdot 3}(T) .
\end{aligned}
$$

From the almost sure uniform convergence of the empirical covariance operators involved in this expression and the equalities

$$
\begin{aligned}
& V_{k \cdot 3} A_{k}^{*} \Pi_{\mathrm{ker}\left(A_{k} V_{k \cdot 3} A_{k}^{*}\right)}=L_{k \cdot 3}^{*} L_{k \cdot 3} A_{k}^{*} \Pi_{\mathrm{ker}\left(L_{k \cdot 3} A_{k}^{*}\right)}=0, \\
& \Pi_{\mathrm{ker}\left(A_{k} V_{k \cdot 3} A_{k}^{*}\right)} A_{k} V_{k j \cdot 3}=\left(V_{j k \cdot 3} A_{k}^{*} \Pi_{\mathrm{ker}\left(A_{k} V_{k \cdot 3} A_{k}^{*}\right)}\right)^{*}=\left(L_{j \cdot 3}^{*} L_{k \cdot 3} A_{k}^{*} \Pi_{\mathrm{ker}\left(L_{k \cdot 3} A_{k}^{*}\right)}\right)^{*}=0,
\end{aligned}
$$


it follows that $\beta_{k \cdot 3}^{(n)}$ converges almost surely uniformly to the operator $\beta_{k \cdot 3}$ defined by

$$
\begin{aligned}
\beta_{k \cdot 3}(T)= & a_{k \cdot 3}(T) A_{k}^{*}\left(A_{k} V_{k \cdot 3} A_{k}^{*}\right)^{\dagger} A_{k} V_{k j \cdot 3} \\
& -V_{k \cdot 3} A_{k}^{*}\left(A_{k} V_{k \cdot 3} A_{k}^{*}\right)^{\dagger} A_{k} a_{k \cdot 3}(T) A_{k}^{*}\left(A_{k} V_{k \cdot 3} A_{k}^{*}\right)^{\dagger} A_{k} V_{k j \cdot 3} \\
& +V_{k \cdot 3} A_{k}^{*}\left(A_{k} V_{k \cdot 3} A_{k}^{*}\right)^{\dagger} A_{k} a_{k j \cdot 3}(T),
\end{aligned}
$$

that is $\mathrm{Z} \beta_{k \cdot 3}=\gamma_{k \cdot 3}+a_{k j \cdot 3}$. Using this later expression and the fact that, under $H_{0}$, the equality $V_{k j \cdot 3}=S_{k \cdot 3}$ is valid, we have:

$$
n C_{k}^{(n)}\left(A_{k}\right)=n\left\|V_{k j \cdot 3}^{(n)}-V_{k j \cdot 3}-\left(S_{k \cdot 3}^{(n)}-V_{k j \cdot 3}\right)\right\|^{2}=\left\|a_{k j \cdot 3}^{(n)}\left(H_{n}\right)-\beta_{k \cdot 3}^{(n)}\left(H_{n}\right)\right\|^{2} .
$$

Thus:

$$
n C^{(n)}\left(A_{1}, A_{2}\right)=N\left(L_{n}\left(H_{n}\right)\right)
$$

where $L_{n}$ is the random operator

$$
u \in M \mapsto\left(\left(\beta_{1 \cdot 3}^{(n)}-a_{12 \cdot 3}^{(n)}\right)(u),\left(\beta_{2 \cdot 3}^{(n)}-a_{21 \cdot 3}^{(n)}\right)(u)\right) \in \mathcal{L}\left(\mathcal{X}_{2}, \mathcal{X}_{1}\right) \times \mathcal{L}\left(\mathcal{X}_{1}, \mathcal{X}_{2}\right)
$$

and $N$ is the continuous map

$$
(v, w) \in \mathcal{L}\left(\mathcal{X}_{2}, \mathcal{X}_{1}\right) \times \mathcal{L}\left(\mathcal{X}_{1}, \mathcal{X}_{2}\right) \mapsto\|v\|^{2}+\|w\|^{2} \in \mathbb{R}
$$

It is easy to verify that $L_{n}$ almost surely uniformly converges to the operator

$$
L: u \in \mathcal{L}\left(\mathcal{X}_{1} \times \mathcal{X}_{2}\right) \mapsto\left(\gamma_{1 \cdot 3}(u), \gamma_{2 \cdot 3}(u)\right) \in \mathcal{L}\left(\mathcal{X}_{2}, \mathcal{X}_{1}\right) \times \mathcal{L}\left(\mathcal{X}_{1}, \mathcal{X}_{2}\right)
$$

Further, we have

$$
\left\|L_{n}\left(H_{n}\right)-L\left(H_{n}\right)\right\|_{\mathcal{L}\left(\mathcal{X}_{2}, \mathcal{X}_{1}\right) \times \mathcal{L}\left(\mathcal{X}_{1}, \mathcal{X}_{2}\right)} \leq\left\|L_{n}-L\right\|_{\infty}\left\|H_{n}\right\|_{M}
$$

since $\left\|H_{n}\right\|_{M}$ (resp. $\left\|L_{n}-L\right\|_{\infty}$ ) converges in distribution (resp. in probability), as $n \rightarrow+\infty$, to $\|H\|_{M}$ (resp. 0 ), this inequality implies the convergence in probability of $L_{n}\left(H_{n}\right)-L\left(H_{n}\right)$ to 0 as $n \rightarrow+\infty$. Hence $L_{n}\left(H_{n}\right)$ and $L\left(H_{n}\right)$ have the same limit distribution; then $L_{n}\left(H_{n}\right)$ likewise converges in distribution to $L(H)$. From the continuity of $N$, it comes that $n C^{(n)}\left(A_{1}, A_{2}\right)$ converges in distribution to $Q=N(L(H))=$ $\left\|\gamma_{1 \cdot 3}(H)\right\|^{2}+\left\|\gamma_{2 \cdot 3}(H)\right\|^{2}$.

Then, for a given (asymptotic) level $\alpha \in] 0,1[$ the null hypothesis is rejected if $n C^{(n)}\left(A_{1}, A_{2}\right)>\mathbb{F}_{Q}^{-1}(\alpha)$, where $\mathbb{F}_{Q}$ denotes the distribution function of $Q$. Notice that since $C^{(n)}\left(A_{1}, A_{2}\right)$ is a strongly consistent estimator of $C\left(A_{1}, A_{2}\right)$ this test is consistent. In practice, one has to replace in equation (4.6) each covariance operator by its estimator introduced in this paper.

Remark 4.2. Additional information hypothesis in canonical analysis was discussed by Fujikoshi (1982) who introduced a likelihood ratio test for this problem. Later, this test was considered by Kariya et al. (1987) in order to test an hypothesis related to selection of variables in the classical MANOVA model, and it was also used by Suzukawa (1997) for evaluating the effect on canonical correlation of imposing linear constraints. This test is mainly based on a normality assumption for the variates. More recently, 
Nkiet (2003) proposed another test for additional information derived from a test for invariance of LCA which does not require to do any assumption on the distribution of the related random variables. A similar approach can be used here for LRCA; indeed, if for $k \in\{1,2\}$ we have the decomposition in direct sum $\mathcal{X}_{k}=\mathcal{X}_{k}^{(1)} \oplus \mathcal{X}_{k}^{(2)}$, a test for additional information in LRCA having the preceding property is obtained by using the test of invariance developed above with $A_{k}:=\pi_{k 1}$, where $\pi_{k 1}$ is the projection operator onto $\mathcal{X}_{k}^{(1)}$ along $\mathcal{X}_{k}^{(2)}$.

\section{Acknowledgements}

The authors are grateful to the referees for their useful remarks and comments which improve the original version of the paper.

\section{REFERENCES}

Anderson, T. W. (1999). Asymptotic theory for canonical correlation analysis, Journal of Multivariate Analysis, 70, 1-29.

Arconte, A. (1980). Étude asymptotique de l'analyse en composantes principales et de l'analyse canonique, Thèse de $3^{\circ}$ cycle, Université de Pau et des Pays de l'Adour.

Baccini, A., Caussinus, H. and Ruiz-Gazen, A. (2001). Apprentissage progressif en analyse discriminante, Revue de Statistique Appliquée, XLIX(4), 87-99.

Cléroux, R. and Lazraq, A. (1988). Étude comparative de différentes mesures de liaison entre deux variables aléatoires vectorielles, Statistique et Analyse des Données, 13, 39-58.

Cramer, E. M. and Nicewander, W. A. (1979). Some symmetric invariant measures of multivariate association, Psychometrika, 41, 347-352.

Dauxois, J. and Nkiet, G. M. (1997a). Canonical analysis of two Euclidean subspaces and its applications, Linear Algebra and Its Applications, 264, 355-388.

Dauxois, J. and Nkiet, G. M. (1997b). Testing for the lack of a linear relationship, Statistics, 30, 1-23.

Dauxois, J. and Nkiet, G. M. (2000). Lois asymptotiques de fonctions des valeurs propres d'une suite d'opérateurs aléatoires autoadjoints, Comptes Rendus de l'Académie des Sciences de Paris, Série $I, \mathbf{3 3 0}, 601-604$.

Dauxois, J. and Nkiet, G. M. (2002). Measures of association for Hilbertian subspaces and some applications, Journal of Multivariate Analysis, 82(2), 263-298.

Dauxois, J. and Pousse, A. (1975). Une extension de l'analyse canonique. Quelques applications, Annales de l'Institut Henri Poincaré, XI, 355-378.

Dauxois, J., Romain, Y. and Viguier, S. (1994). Tensor products and Statistics, Linear Algebra and Its Applications, 210, 59-88.

Dauxois, J., Nkiet, G. M. and Romain, Y. (2004). Canonical analysis relative to a closed subspace, 10th Special Issue in Statistics, Linear Algebra and Its Applications (to appear).

Dossou-Gbete, S. and Pousse, A. (1991). Asymptotic study of eigenelements of a sequence of random selfadjoint operators, Statistics, 22, 479-491.

Eaton, M. L. and Tyler, D. (1994). The asymptotic distribution of singular values with applications to canonical correlations and correspondence analysis, Journal of Multivariate Analysis, 50, 238-264.

Fine, J. (2000). Étude asymptotique de l'analyse canonique, Publications de l'Institut de Statistique de l'Université de Paris, 44(2-3), 21-72.

Fujikoshi, Y. (1982). A test for additional information in canonical correlation analysis, Annals of the Institute of Statistical Mathematics, 34, 523-530.

Fujikoshi, Y. and Khatri, C. G. (1990). A study of redundancy of some variables in covariate discriminant analysis, Annals of the Institute of Statistical Mathematics, 42, 769-782.

Fujikoshi, Y. and Veitch, L. G. (1979). Estimation of dimensionality in canonical correlation analysis, Biometrika, 66(2), 345-352.

Hsu, P. L. (1941). On the limiting distribution of the canonical correlation, Biometrika, 32, 38-45. 
Kariya, T., Fujikoshi, Y. and Krishnaiah, P. R. (1987). On tests for selection of variables and independence under multivariate regression models, Journal of Multivariate Analysis, 21, 207-237.

Larrère, G. (1994). Contributions à l'étude asymptotique en analyse multivariée, Thèse de doctorat, Université de Pau et des Pays de l'Adour.

Lin, P. E. (1987). Measures of association between vectors, Communication in Statistics-Theory Methods, 16(2), 321-338.

Mathai, A. M. and Provost, S. B. (1992). Quadratic Forms in Random Variables. Theory and Methods, Dekker, New York.

Muirhead, R. J. and Waternaux, C. M. (1980). Asymptotic distributions in canonical analysis and other multivariate procedures for nonnormal populations, Biometrika, 67(1), 31-43.

Nashed, M. Z. (1976). Perturbation and approximations for generalized inverses and linear operator equations, Generalized Inverses and Applications (ed. M. Z. Nashed), 325-396, Academic Press, New York.

Nkiet, G. M. (2000). Comparaison de tests de non corrélation, Annales des Sciences Mathématiques du Québec, 24(2), 155-178.

Nkiet, G. M. (2003). Inference for the invariance of canonical analysis under linear transformations, Journal of Multivariate Analysis, 84(1), 1-18.

Pousse, A. (1992). Études asymptotiques, Modèles pour l'analyse des données multidimensionnelles (eds. J. J. Drosbeke, B. Fichet and P. Tassi), Economica, Paris.

Rao, B. Raja (1969). Partial canonical correlations, Trabajos de Estadística y de Investigación Operativa, 20, 211-219.

Siotani, M. (1957). Effect of the additional variates on the canonical correlation coefficients, Proceedings of the Institute of Statistical Mathematics, 5, 52-57.

Suzukawa, A. (1997). Statistical inference in a canonical correlation analysis with linear constraints, Journal of the Japan Statistical Society, 27(1), 93-107.

Suzukawa, A. and Sato, Y. (1996). The non-null distribution of the likelihood ratio criterion for additional information hypothesis in canonical correlation analysis, Journal of the Japan Statistical Society, 26(1), 91-100.

Timm, N. H. and Carlson, J. E. (1976). Part and bipartial canonical correlation analysis, Psychometrika, 41(2), 159-176.

Yanai, H. and Takane, Y. (1992), Canonical correlation analysis with linear constraints, Linear Algebra and Its Applications, 176, 75-89. 